\title{
A Practical Model for Fluid Flow in Discrete-Fracture Porous Media by Using the Numerical Manifold Method
}

\author{
Mengsu $\mathrm{Hu}^{1,2}$ \\ ${ }^{1}$ College of Civil and Transportation Engineering, Hohai University, \\ Nanjing, 210098, China \\ ${ }^{2}$ Energy Geosciences Division, Lawrence Berkeley National Laboratory, \\ Berkeley, CA 94720, USA \\ Email address: mengsuhu@lbl.gov \\ Telephone Number: 1-510-486-5154 \\ Jonny Rutquist ${ }^{2}$ \\ ${ }^{2}$ Energy Geosciences Division, Lawrence Berkeley National Laboratory, \\ Address: 1 Cyclotron Rd., MS74R316C, Berkeley, CA 94720, USA \\ Email address: jrutqvist@lbl.gov \\ Telephone Number: 1-510-486-5432 \\ Yuan Wang ${ }^{1,2, *}$ ( Corresponding author) \\ ${ }^{1}$ College of Civil and Transportation Engineering, Hohai University, \\ Nanjing, 210098, China \\ ${ }^{2}$ Energy Geosciences Division, Lawrence Berkeley National Laboratory, \\ Berkeley, CA 94720, USA \\ Email address: wangyuanhhu@163.com
}




\title{
A Practical Model for Fluid Flow in Discrete-Fracture Porous
}

\section{Media by Using the Numerical Manifold Method}

\author{
Mengsu $\mathrm{Hu}^{1,2}$, Jonny Rutqvist ${ }^{2}$, Yuan Wang ${ }^{1,2, *}$ \\ ${ }^{1}$ College of Civil and Transportation Engineering, Hohai University, \\ Nanjing, 210098, China \\ ${ }^{2}$ Energy Geosciences Division, Lawrence Berkeley National Laboratory, Berkeley, \\ CA 94720, USA
}

\begin{abstract}
In this study, a numerical manifold method (NMM) model is developed to analyze flow in porous media with discrete fractures in a non-conforming mesh. This new model is based on a two-cover-mesh system with a uniform triangular mathematical mesh and boundary/fracture-divided physical covers, where local independent cover functions are defined. The overlapping parts of the physical covers are elements where the global approximation is defined by the weighted average of the physical cover functions. The mesh is generated by a tree-cutting algorithm. A new model that does not introduce additional degrees of freedom (DOF) for fractures was developed for fluid flow in fractures. The fracture surfaces that belong to different physical covers are used to represent fracture flow in the direction of the fractures. In the direction normal to the fractures, the fracture surfaces are regarded as Dirichlet boundaries to exchange fluxes with the rock matrix. Furthermore, fractures that intersect with Dirichlet or Neumann boundaries are considered. Simulation examples are designed to verify the efficiency of the tree-cutting algorithm, the calculation's independency from the mesh orientation, and accuracy when modeling porous media that contain fractures with multiple intersections and different orientations. The simulation results show good agreement with available analytical solutions. Finally, the model is applied to cases that involve 9 intersecting fractures and a complex network of 100 fractures, both of which achieve reasonable results. The new model is very practical for modeling flow in fractured porous media, even for a geometrically complex fracture network with large hydraulic conductivity contrasts between fractures and the matrix.
\end{abstract}

Keywords: fluid flow, discrete fractures, porous media, non-conforming mesh, numerical manifold method

\section{Introduction}

Fractures are ubiquitous in natural geological porous media as products of folding, faulting, subsidence, thermal deformation, or other geological activities over time. Fractures may act as conduits or seals for fluid flow, and their size ranges from 
microns to kilometers. Analyzing flow in naturally fractured porous media is crucial in a wide range of geological and environmental engineering applications, including hydrocarbon and geothermal reservoir engineering, nuclear waste disposal, CO2 sequestration, and ground water management [1-3].

The methods to simulate flow in fractures are categorized into the following types depending on the heterogeneity of the fractured porous media: (1) equivalent continuum models, (2) discrete fracture models, (3) their hybrid [1, 4-7] or (4) dual-continuum models [8-9]. In the aforementioned applications, the presence of arbitrarily oriented and intersecting fractures may influence the flow in a complex manner that cannot be simplified with homogenization. Therefore, modeling flow in discrete fractures is very important. Among existing discrete fracture models, two approaches have been developed that depend on the permeability of rock matrix. If the permeability of the rock matrix is negligible, fluid flow is assumed to only occur within a connected fracture network [10-14]. The other approach considers fluid flow in both the rock matrix and fractures, i.e. the discrete-fracture-matrix model, in which the rock matrix may act the primary medium for flow when the fractures are sealed. In this study, we focus on the modeling of fluid flow in discrete fractured porous media in $2 \mathrm{D}$ by considering a permeable rock matrix as porous media.

The greatest challenges of modeling fluid flow in discretely fractured porous media are the complex geometry of fractures, the large contrasts in geometries and the hydraulic properties between fractures and the rock matrix. One approach to model such a problem is to use the same dimension for fractures as for the rock matrix in an equi-dimensional formulation [15-20]. The authors developed a similar approach for dominant fractures as a heterogeneous medium [21]. When fractures densely intersect, high computational load and convergence problems may appear because of small integration areas. Another formulation that is based on the concept of reduced dimension has been proposed to avoid such issues [22-34]. The basic idea of a reduced-dimension formulation is to represent the fractures with an $n-1$ dimensional mesh in an $n$-dimensional problem. A variety of numerical methods have been developed based on the reduced-dimension idea, including finite difference, finite volume and finite element methods [22-32]. In these models, the fractures are usually represented in a mesh by interfaces between matrix elements, which requires that the mesh conforms to the fractures. For example, a series of $3 \mathrm{D}$ finite-volume models was applied to analyze well tests [27], single-phase [28-29], two-phase [30], and transient compressible flow [31-32] under the condition that a robust meshing algorithm is provided. Non-conforming-mesh methods for fluid analysis in fracture-matrix systems, in which the mesh does not have to conform to the fracture geometries, were developed based on mixed [33] and extended [34] finite element methods. However, in such an extended finite element formulation, an enriched approximation of fractures may limit the applicability of modeling complexly intersecting fractures, similar to problems that are related to mechanical analysis, as discussed by An et al. [35]. Therefore, an improved non-conforming-mesh method that can achieve high accuracy and deal with complexly intersecting fractures is required. 
The numerical manifold method (NMM) is a promising numerical method for modeling mechanical processes in continuous/discontinuous media [36-37]. The establishment of the NMM was motivated by the urgent need to analyze dynamic processes that span both continuous and discontinuous media in a unified approach, which is not possible with continuous methods such as the finite element method (FEM) or discontinuous methods such as the distinct element method. The NMM has indeed been successfully applied to rock mechanics analyses of both continuous and discontinuous geologic media [38-40]. The numerical grids (or meshes) in the NMM consist of mathematical and physical covers. Mathematical covers overlay the entire material domain and determine the approximation's precision by the mesh density. The physical covers are divided from the mathematical covers by boundaries and discontinuities (such as fractures) and determine the integration domain. The overlapping parts of physical covers form elements. The generation of physical covers and elements can be conveniently realized by a one-time tree-cutting algorithm [41]. Each of the divided physical covers has its independent local function. The global approximation of an element is the weighted average of the cover function that overlaps the element. Based on this definition framework, the NMM appears to be a promising method for modeling flow in discontinuous media and superior to continuous methods. Specifically, the following features of the NMM can be highlighted as particularly advantageous for solving discontinuous/continuous fluid flow problems: (1) the meshing effort can be substantially reduced with non-conforming mathematical covers; (2) the physical covers that are divided by a fracture have independent functions, which represent the discontinuous nature of the fractures and do not require enrichment functions; (3) the intersection of fractures can be realized by a tree-cutting algorithm and explicitly represented by divided physical covers and elements; and (4) the simplex integration that is used in the NMM [37] achieves exact solutions in polygons with complex shapes. For fluid flow modeling using NMM, Ohnishi et al. [42] developed a node-shifting method for saturated-unsaturated flow modeling with the NMM. In [21], the authors compared two methods for modeling heterogeneous flow, including flow in equi-dimensional fractures based on an energy-work seepage model [43]. Recently, a mixed head- flux formulation was also proposed and applied to unconfined fluid flow in porous media [44].

In this study, we develop a new model to analyze flow in porous media that contain discrete fractures by using the NMM with a non-conforming mesh. First, we introduce the fundamentals of the NMM in Section 2, including the fundamental definitions, the global approximation, and the tree-cutting algorithm to generate the mesh from a given discrete fracture network geometry. In Section 3, we present the development of the new model for flow along and normal to fractures without introducing additional degrees of freedom for the fractures. In Section 4, we verify the new model with some examples and compare the results to the analytical solutions. In Section 5, the new model is applied to two problems with densely intersecting fractures. In Section 6, we discuss some potential extensions of the current model to 3D fluid flow analysis and to coupled hydro-mechanical analysis for 
discrete-fracture porous media.

\section{Fundamentals of the NMM}

In this section, we introduce the fundamentals of the NMM, including the NMM's approximation for fluid flow, the relationship of the NMM to other methods such as the finite element method (FEM) and distinct deformation analysis (DDA), and the generation of discrete fracture with a tree-cutting algorithm.

\subsection{NMM approximation}

The NMM, which was proposed by Shi [36], is based on the concept of "manifold" in mathematical topology. In the NMM, independent mathematical and physical covers are defined. The mathematical covers are user-defined and consist of finite overlapping covers that occupy the entire material domain. Regular meshes such as finite difference grids, finite elements or convergence regions of series can be used as mathematical covers. These mathematical covers determine the numerical precision by the mesh shape and mesh density. For example, equilateral triangular mathematical covers perform better than rectangular covers in terms of the precision and stability of the solution, as derived and proven by examples in 2D mechanical analysis [45]. On the other hand, the numerical precision increases with a denser approximation mesh (i.e. the mathematical mesh in the NMM) to a certain extent. The physical covers are mathematical covers that are divided by boundaries, fractures or discontinuities, which determine the integration fields. A local approximation is assigned on each physical cover, for example, a constant (simplified, as in the FEM), linear function or series. The weighted average of the local cover functions forms the global approximation. Therefore, the NMM is flexible and general enough to include and combine well-developed analytical methods, the widely used FEM, and block-oriented discontinuous deformation analysis (DDA) in a unified form.
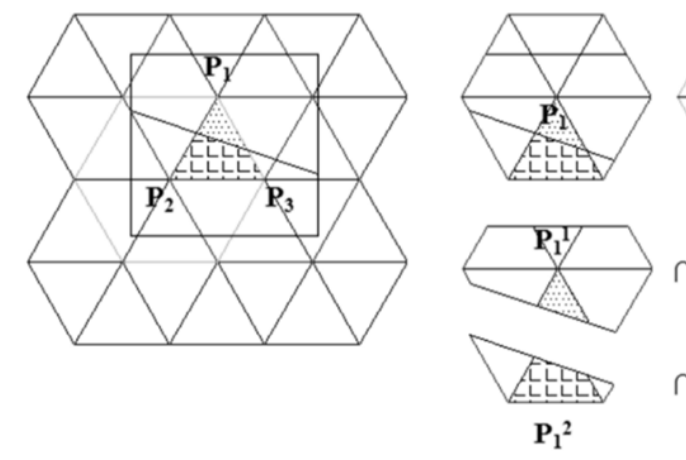
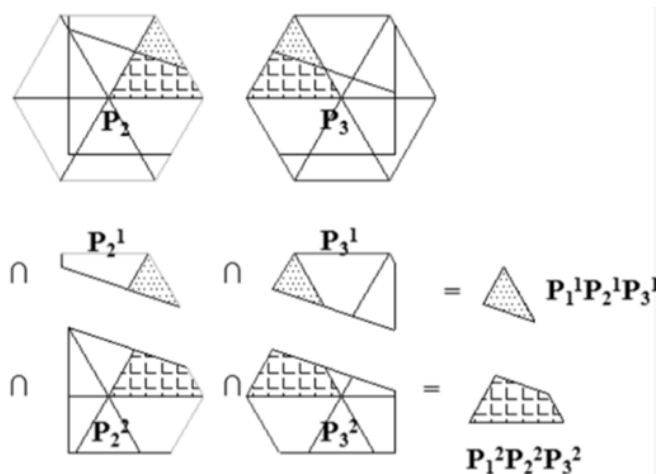

Figure 1 Schematic of a fractured domain that was discretized in the NMM

Here, we briefly introduce the NMM approximation that we use to model fluid flow in discrete-fracture porous media. For a 2D problem, simplexes such as triangles or rectangles are usually used to form the mathematical covers. Here, we use triangular elements for the mathematical covers. Figure 1 shows a schematic of a 
fractured domain that was discretized in the $\mathrm{NMM}$, including the mathematical covers, physical covers and elements. As shown, all the triangles that contain a certain mesh vertex (designated with stars) form a mathematical cover (i.e., a hexagon such as $\mathrm{P}_{1}, \mathrm{P}_{2}$, and $\left.\mathrm{P}_{3}\right)$. The corresponding physical covers $\left(\mathrm{P}_{1}{ }^{1}\right.$ and $\mathrm{P}_{1}{ }^{2}, \mathrm{P}_{2}{ }^{1}$ and $\mathrm{P}_{2}{ }^{2}$, and $\mathrm{P}_{3}{ }^{1}$ and $\mathrm{P}_{3}{ }^{2}$ ) are divided from the mathematical covers by the boundaries or discontinuities. The overlapping areas of the physical covers are defined as elements (such as $\mathrm{P}_{1}{ }^{1} \mathrm{P}_{2}{ }^{1} \mathrm{P}_{3}{ }^{1}$ and $\mathrm{P}_{1}{ }^{2} \mathrm{P}_{2}{ }^{2} \mathrm{P}_{3}{ }^{2}$, which are distinguished by different shadow patterns). Figure 1 shows that the physical covers and elements are a non-conforming mesh. Moreover, both continuous and discontinuous behavior can be modeled once a mathematical cover is divided into different physical covers [36, 38-40].

The field variable $\varphi$ at a point $(x, y)$ in element $e$ is the weighted average of the functions of all the physical covers that overlap the element:

$$
\varphi(x, y)=\sum_{i=1}^{N_{p c}^{(e)}} w_{i}(x, y) \varphi_{i}(x, y)
$$

where $N_{p c}{ }^{(e)}$ is the number of physical covers that are related to element $e, \varphi_{i}(x, y)$ is the hydraulic head function of the physical cover $i$, and $w_{i}(x, y)$ is the weight function of the physical cover $i$ on element $e$. Here, the field variable for fluid flow analysis is the hydraulic head $h$. Each physical cover $i$ satisfies

$$
\begin{cases}w_{i}(x, y)>0 & (x, y) \in U_{i} \\ w_{i}(x, y)=0 & (x, y) \notin U_{i}\end{cases}
$$

where $U_{i}$ is the geometric range of the physical cover $i$.

The cover function $\varphi_{i}(x, y)$ of the physical cover $i$ can be a constant, linear function or locally defined series:

$$
\varphi_{i}(x, y)=\sum_{j=1}^{m} s_{j} \varphi_{i j}=\left(\begin{array}{lllll}
1 & x & y & \cdots & s_{m}
\end{array}\right)\left(\begin{array}{c}
\varphi_{i 1} \\
\varphi_{i 2} \\
\varphi_{i 3} \\
\vdots \\
\varphi_{i m}
\end{array}\right)
$$

where $m$ and $\varphi_{i j}$ are the number of degrees of freedom (DOF) and the $j$ th DOF of the physical cover $i$, respectively; and $s_{j}$ is the coefficient of the $j$ th DOF. For a complete Nth-order physical cover function, $m=(N+1)(N+2) / 2$. In this paper, we use linear weight functions and constant physical cover functions $h_{i}(x, y)(m=1)$ on the triangular mesh for fluid flow analysis.

\subsection{Relationship among the NMM, FEM and DDA}

The FEM and DDA are numerical methods that have been applied to model fluid flow in porous and/or fractured media. Here, we show that the FEM and DDA can actually be considered as simplified subsidiaries of the NMM. Let us consider two special cases of the FEM and DDA as subsidiary simplifications of the NMM.

The FEM can be considered a simplification of the NMM if the cover functions 
that were defined in Equation (3) are constants, the weight functions are formed by shape functions, each integral area is exactly the area of the mathematical mesh, and no discontinuities are included. Compared to the FEM, the NMM has the following advantages: (1) the ability to deal with discontinuities. In the FEM, the mesh vertices should be aligned on the boundaries and interface elements should be introduced to represent the discontinuities, which make analyzing intersecting fractures as discontinuities very cumbersome. However, in the NMM, different physical covers that are divided by discontinuities can represent discontinuous features across these discontinuities. This advantage has previously been utilized to analyze discontinuous mechanical processes [36-40] and will be explored in this study to analyze fluid flow in porous rock masses with discrete fractures; (2) independent approximation fields (consisting of a regular mathematical mesh) and integral fields (consisting of physical covers that are divided by boundaries and discontinuities), which dramatically reduce meshing efforts; and (3) flexibility when enhancing approximations. In the FEM, the so-called $h$-adaptive and $p$-adaptive concepts were proposed to enhance the precision of approximations. The $\mathrm{H}$-adaptive $\mathrm{FEM}$ can be very computationally expensive for complex-geometry and high-gradient problems, while the p-adaptive FEM leads to incomplete approximations. However, the approximation precision in the NMM can be enhanced by increasing the order of the cover functions, as in Equation (3).

DDA can be considered a simplification of the NMM when modeling discontinuous media with blocks that formed from fractures. If these blocks are divided by natural discontinuities form physical covers (mathematical covers and physical covers are the same in this case) and no overlapping areas exist among these physical covers,

$$
w_{i}(x, y)=1
$$

and cover functions (as displacements) for 2D mechanical analysis are defined as follows:

$$
\left\{\begin{array}{l}
u_{i}(x, y) \\
v_{i}(x, y)
\end{array}\right\}=\left(\begin{array}{cccccc}
1 & 0-y-y_{0} & x \rightarrow x_{0} & (\quad) & y-y_{0} & 0 \\
0 & 1 & x-x_{0} & (\quad) & y-y_{0} & 0 x-x_{0}
\end{array}\right)\left\{\begin{array}{l}
u_{0} \\
v_{0} \\
r_{0} \\
\varepsilon_{x} \\
\varepsilon_{y} \\
\gamma_{x y}
\end{array}\right\}
$$

The NMM is simplified as DDA. If the DOFs that were defined on these blocks are only related to the inter-flux hydraulically or inter-forces mechanically from neighboring blocks (which may not be deformable), the NMM is simplified as discrete methods, such as the DEM, or polygonal-element-based methods, such as the finite volume method (FVM) for fluid flow analysis or rigid body spring network (RBSN) for mechanical analysis. Compared to the DDA and DEM methods, the NMM is advantageous because of its ability to deal with discontinuous and continuous 
problems in a unified manner.

A more thorough comparison between the NMM and XFEM for mechanical discontinuous analysis was presented by An et al. [35].

\subsection{Discrete-fractures-contained mesh generation by tree cutting}

In order to generate the discrete-fractures-contained non-conforming mesh in NMM, we use a "tree cutting" algorithm developed by Shi [41]. By tree cutting, the intersecting discrete fractures and boundaries divide the mathematical covers into physical covers and thereafter form corresponding elements. Here, we briefly introduce the basic idea of tree cutting.

Originally developed for impermeable rock matrices, the basic idea of a tree-cutting algorithm is to delete all the fractures that are not fluid conductive in a network loop, similar to cutting trees, and to keep fluid-conductive fractures. First, we demonstrate a case where the rock matrix is impermeable and no discretization mesh is introduced, as shown in Figure 2. In this rectangular domain, multiple intersecting fractures are represented by solid lines, which are shown as "Original" in Figure 2. We numbered the boundaries and fractures from 1 to 10 . To generate the fracture network, the fractures that do not connect to other boundaries or fractures are assumed not to be fluid conductive and are deleted. Fractures with only one intersection, including fractures 9 and 11, are deleted first, which is shown as " 1 st -round tree cutting" in Figure 2. After deleting these fractures, other fractures may have only one intersection, becoming nonconductive, and are also deleted, such as fracture 8, which is shown as " ${ }^{\text {nd }}$-round tree cutting" in Figure 2. This tree cutting is repeated until all the fractures have at least two intersections, meaning that they are conductive, which is shown as "Final" in Figure 2. At the same time, independent elements (defined as blocks in DDA) are generated as polygons that are bounded by these fractures.
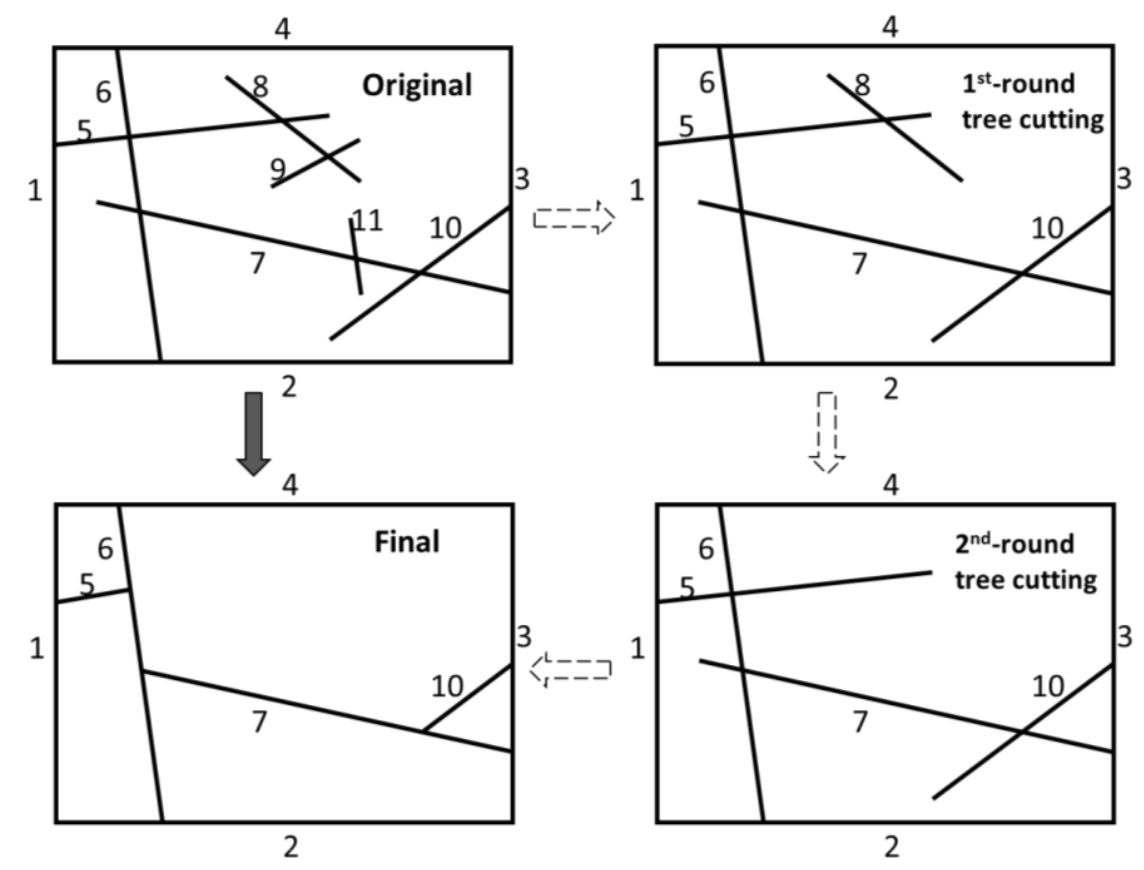
Figure $\mathbf{2}$ Schematic of tree cutting to generate a fracture network in an impermeable rock matrix without a discretization mesh

To realize the tree-cutting algorithm in the code, we use a matrix with fracture numbers to record the connectivity of fractures/boundaries during tree cutting. For example, line 1 is originally connected to lines 2,4 and 5 . After recording all the information for the original connectivity, we delete the rows with only one number for each, which represent only one intersection, such as the $9^{\text {th }}$ and $11^{\text {th }}$ rows. Then, we delete other rows with only one number for each, such as the $8^{\text {th }}$ row. Once all the rows have at least two numbers, we obtain the final result for the generated fracture network, which consists of intersecting, flow-conducting fractures and boundaries.

Table 1 Connectivity matrix of fractures/boundaries in tree cutting

\begin{tabular}{l|lll}
\hline $\begin{array}{c}\text { Boundaries } \\
\text { /Fractures }\end{array}$ & $\begin{array}{l}\text { Originally connected } \\
\text { fractures/boundaries }\end{array}$ & $\begin{array}{l}\text { 1st-round } \\
\text { tree cutting }\end{array}$ & $\begin{array}{l}\text { 2nd-round } \\
\text { tree cutting }\end{array}$ \\
\hline $\mathbf{1}$ & $2,4,5$ & $2,4,5$ & $2,4,5$ \\
$\mathbf{2}$ & $1,3,6$ & $1,3,6$ & $1,3,6$ \\
$\mathbf{3}$ & $2,4,7,10$ & $2,4,7,10$ & $2,4,7,10$ \\
$\mathbf{4}$ & $1,3,6$ & $1,3,6$ & $1,3,6$ \\
$\mathbf{5}$ & $1,6,8$ & $1,6,8$ & 1,6 \\
$\mathbf{6}$ & $2,4,5,7$ & $2,4,5,7$ & $2,4,5,7$ \\
$\mathbf{7}$ & $3,6,10,11$ & $3,6,10$ & $3,6,10$ \\
$\mathbf{8}$ & 5,9 & 5 & \\
$\mathbf{9}$ & 8 & & \\
$\mathbf{1 0}$ & 3,7 & 3,7 & 3,7 \\
$\mathbf{1 1}$ & 7 & & \\
\hline
\end{tabular}

Original triangular mesh and physical domain containing discrete fractures
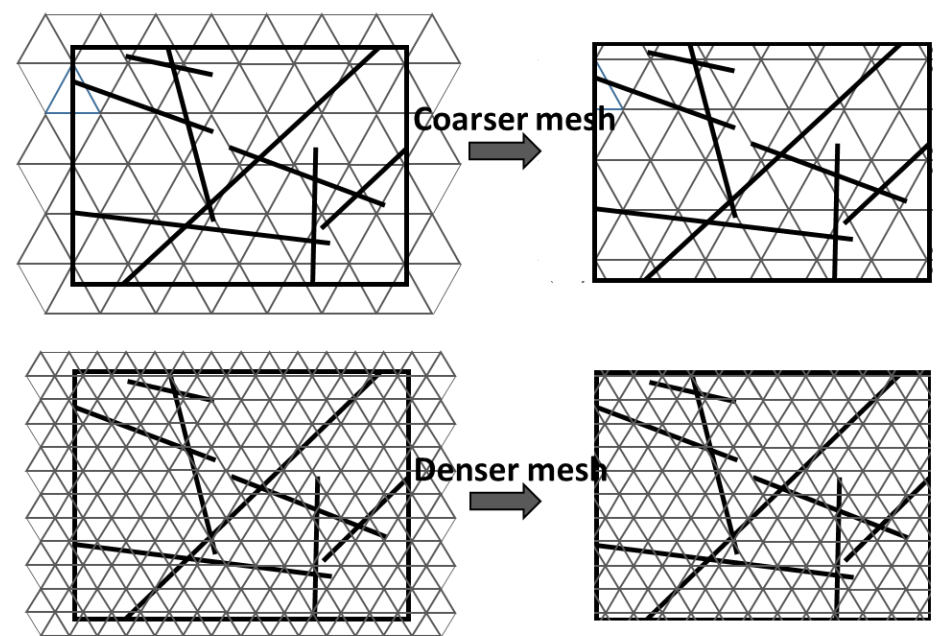

Generated mesh with the second method to represent fractures

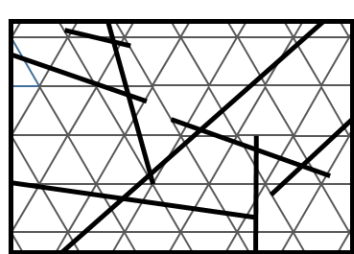

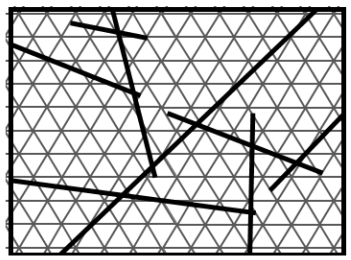


Figure 3 Generated mesh by tree cutting with coarser and denser triangular meshes with the two alternative methods to represent fractures

Now, we consider that the rock matrix is permeable and exchanges flux with fractures. In this case, a discretization mesh is required for the rock matrix, which differs from the case with an impermeable rock matrix. Therefore, all the mathematical mesh lines, fractures and boundaries should be considered in the tree cutting to generate the physical covers and elements. Here, we use a triangular mathematical mesh to overlap the entire simulation domain. Once the mathematical mesh is established, we can use the same tree-cutting algorithm and code to generate the physical covers and elements, which are divided by fractures, boundaries, and the edges of the triangular mesh. However, another important difference from the impermeable-rock-matrix case is that fractures with no or only one intersection with other fractures are still fluid conductive and should not be deleted. Two possible alternatives exist to preserve every fracture. One possibility is to use two coinciding lines to represent one fracture, which originally have more than one intersection and will not be deleted automatically. Another possibility is to represent each fracture with a single line while using a denser mesh as an approximation. In such an algorithm, fracture segments with no or only one intersection with other fractures, boundaries or mesh edges will still be deleted. However, denser meshes have more intersections with these fractures, making the deleted segments negligible. One of these two alternative methods is chosen depending on the density of the fractures and the number of fractures with no or only one intersection. Physical covers and elements can be generated by using the tree-cutting algorithm with these two alternative methods for representing fractures, as shown in Figures 3 and 4. Interestingly, fractures are regarded as discontinuities between elements and thus do not influence the approximation field if the fractures are impermeable. Therefore, we can readily model cases of fluid flow in porous media with sealed fractures with the tree-cutting algorithm to generate a mesh and scheme for modeling fluid flow in porous media, as demonstrated in Section 4.1.

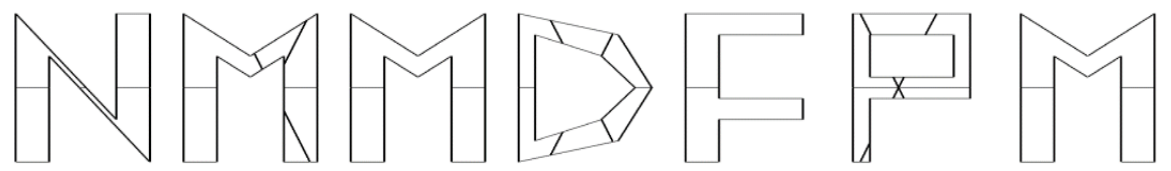

Figure 4 Generated mesh in an NMM triangular mesh by tree cutting

\section{New model for fracture fluid flow}

We can generate physical covers and elements in the NMM that are divided by discrete fractures, boundaries and triangular mesh lines by using the tree-cutting algorithm. In such a mesh, fractures are represented by the surfaces of the divided physical covers. Fluid flow in the porous media, which satisfies Darcy's law and mass conservation, is modeled based on an energy-work seepage model with a Lagrange multiplier method for the boundaries (developed by the authors in [21]). Therefore, 
we mainly introduce the development of a new model for fracture flow in this paper.

In 2D analysis, fluid flow in fractures should be considered in two directions: flow along the fractures, which satisfies the cubic law, and flow normal to the surfaces of the fractures as flux exchange with the surrounding rock matrix, as shown in Figure 5. To simulate this behavior, $\mathrm{n}-1$ dimensional models were developed [22-34] by introducing additional 1D fracture elements for 2D analysis. However, the introduced 1D elements and their corresponding DOFs make establishing global equilibrium equations problematic, especially when new fracture creation (fracturing of the matrix) is considered. To overcome this problem, we develop a new model that can model along-fracture and normal-to-fracture flow with no need to introduce additional DOFs for the fractures.
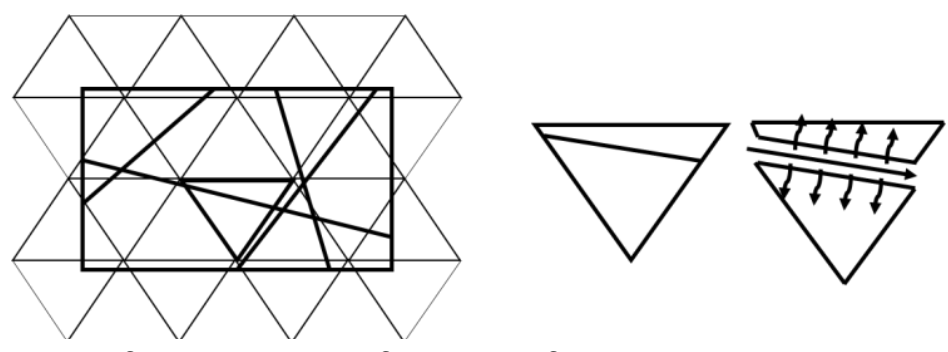

Figure 5 Schematic of a new model for along-fracture and normal-to-fracture flow

\subsection{Flow along fractures}

Fluid flow in fractures satisfies Darcy's law:

$$
\left(\begin{array}{c}
v_{s}^{f} \\
v_{n}^{f}
\end{array}\right)=-\left(\begin{array}{ll}
k_{f} & 0 \\
0 & k_{f n}
\end{array}\right)\left(\begin{array}{c}
\frac{\partial h^{f}}{\partial s} \\
\frac{\partial h^{f}}{\partial n}
\end{array}\right)
$$

where $v$ is the specific discharge; $s$ and $n$ denote the directions along and normal to the fractures, respectively; and $k_{f}$ and $k_{f n}$ are the hydraulic conductivities along and normal to the fracture, respectively. $k_{f}$ can be defined according to Witherspoon et al. [46] depending on the size of interconnected voids between the two fracture surfaces and the hydraulic fracture aperture $b_{h}$ :

$$
k_{f}=\frac{b_{h}^{2} \rho_{f} g}{12 \mu_{f}}
$$

where $\rho_{f}$ and $\mu_{f}$ are the fluid density and dynamic viscosity, and $g$ is the gravitational acceleration. 


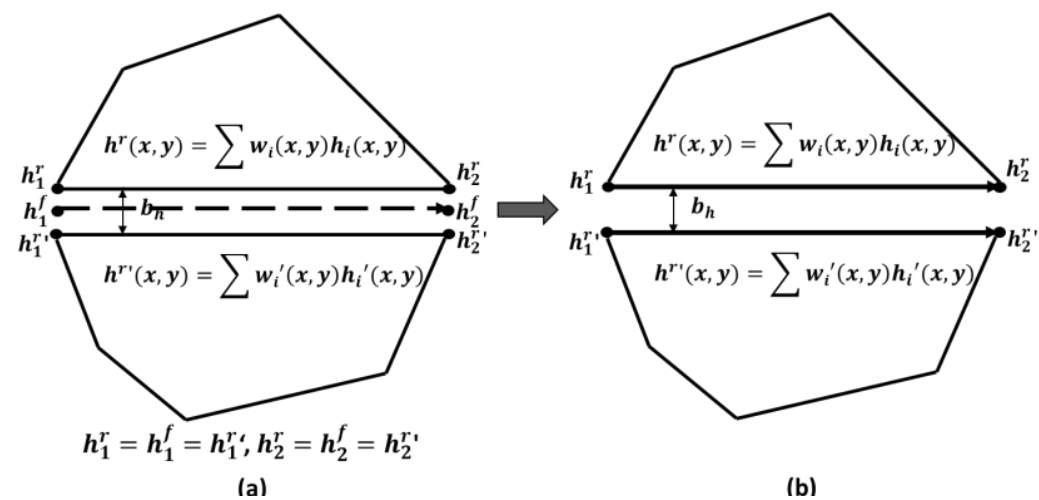

Figure 6 New approach for along-fracture flow without introducing additional DOFs for fractures

Figure 6a shows fluid flow along fractures that is driven by the hydraulic head difference from $h_{1}^{f}$ to $h_{2}^{f}$, which satisfies Darcy's law according to Equations (4) and (5). Because the studied fractures are very thin (microns to millimeters),

$$
h_{1}^{r}=h_{1}^{f}=h_{1}^{r^{\prime}}, \quad h_{2}^{r}=h_{2}^{f}=h_{2}^{r^{\prime}}
$$

where $h^{r}$ and $\mathrm{h}^{\mathrm{r}_{1}}$ denote the hydraulic head on different elements that are divided by the fracture. The hydraulic head within the fracture is uniform across its thickness, so we assume that the fluid flow along a very thin fracture is represented by flow along its two surfaces:

$$
v_{s}^{f}=-k_{f} \frac{\partial h^{r}}{\partial s}=-k_{f} \frac{\partial h^{r^{\prime}}}{\partial s}
$$

The two surfaces belong to different physical covers and elements. According to the energy-work seepage model that was developed in [43], the work that is associated with flow along a fracture is the summation of the work that is associated with flow along these two surfaces:

$$
W_{s}^{f}=\frac{1}{2} \gamma b_{h}\left(\int_{\Gamma} v_{s}^{f} \frac{\partial h^{r}}{\partial s} d s+\int_{\Gamma} v_{s}^{f} \frac{\partial h^{r^{\prime}}}{\partial s} d s\right)
$$

According to Equation (4), the hydraulic gradient along each fracture segment is a constant. Therefore,

$$
\begin{aligned}
& v_{s}^{f} \frac{\partial h^{r}}{\partial s}=-k_{f}\left(\frac{\partial h^{r}}{\partial s}\right)^{2}=-k_{f} \frac{\left(h_{1}^{r}-h_{2}^{r}\right)^{2}}{l^{2}} \\
& v_{s}^{f} \frac{\partial h^{r^{\prime}}}{\partial s}=-k_{f}\left(\frac{\partial h^{r^{\prime}}}{\partial s}\right)^{2}=-k_{f} \frac{\left(h_{1}^{r^{\prime}}-h_{2}^{r^{\prime}}\right)^{2}}{l^{2}}
\end{aligned}
$$

where $l=\left(x_{1}-x_{2}\right)^{2}+\left(y_{1}-y_{2}\right)^{2}$ is the length of the fracture segments. Combined with Equations (1)-(3), 


$$
\begin{aligned}
W_{s}^{f} & =-\frac{1}{2} \gamma b_{h} k_{f} \frac{\left[\sum w_{i}\left(x_{1}, y_{1}\right) h_{i}\left(x_{1}, y_{1}\right)-\sum w_{i}\left(x_{2}, y_{2}\right) h_{i}\left(x_{2}, y_{2}\right)\right]^{2}}{l^{2}}\left[\left(x_{1}-x_{2}\right) \cos \theta+\left(y_{1}-y_{2}\right) \sin \theta\right] \\
& -\frac{1}{2} \gamma b_{h} k_{f} \frac{\left[\sum w_{i}^{\prime}\left(x_{1}, y_{1}\right) h_{i}^{\prime}\left(x_{1}, y_{1}\right)-\sum w_{i}^{\prime}\left(x_{2}, y_{2}\right) h_{i}^{\prime}\left(x_{2}, y_{2}\right)\right]^{2}}{l^{2}}\left[\left(x_{1}-x_{2}\right) \cos \theta+\left(y_{1}-y_{2}\right) \sin \theta\right]
\end{aligned}
$$

where $\cos \theta$ and $\sin \theta$ represent the orientation of the fracture segment. According to the energy-work theorem and the minimization of the total potential energy, we can obtain the final terms in the global equilibrium equations that are associated with along-fracture flow, as shown in Section 3.4.

We calculate a simple $2 \mathrm{~m} \times 2 \mathrm{~m}$ square rock domain that contains a single horizontal fracture (Figure $7 a$ ) to verify this modeling of flow along fractures. The fracture intersects with the upstream and downstream boundaries with hydraulic heads of $10 \mathrm{~m}$ and $0 \mathrm{~m}$, respectively. The permeability contrast between the fracture and rock matrix is 2000, and the aperture of the fracture is $1 \mathrm{~mm}$. This problem involves along-fracture flow together with a Dirichlet boundary condition for fractures (introduced in Section 3.3). We simulate this problem to verify the along-fracture component because we cannot find other simple examples that only involve along-fracture flow. For such an example, we do not need to activate the subroutine for the fracture-matrix flux exchange analysis (introduced in Section 3.2). This scenario is equivalent to a problem of fluid flow in a single-fracture-included heterogeneous media with three vertically different types of material (including the fracture), which satisfies Darcy's law. Therefore, the analytical solution is $h=10-5 x$. We use a minimum mesh with 13 elements and 15 DOFs for the calculation and achieve the hydraulic head gradient of the analytical solution, as shown in Figure 7. The results show the high accuracy and efficiency of the new model for along-fracture flow with no need to introduce additional DOFs for the fractures.

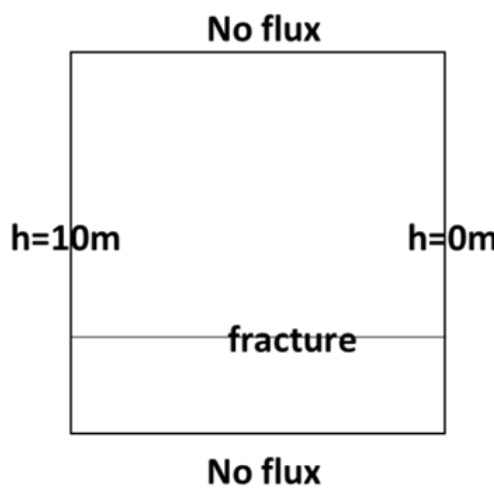

(a)

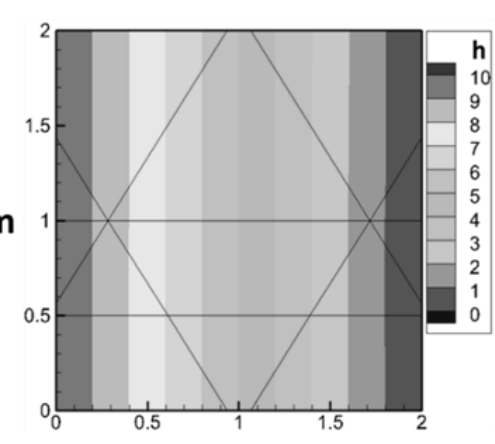

(b)

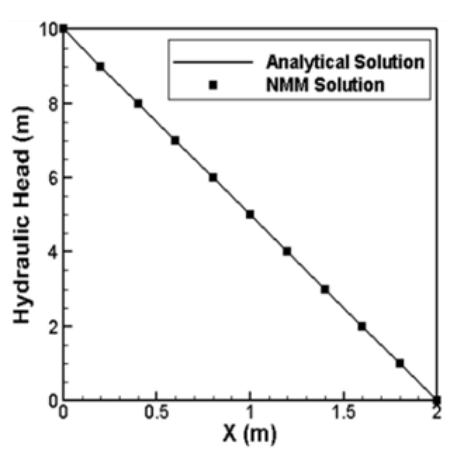

(c)

Figure 7 Verification of along-fracture flow: (a) the geometry and boundary condition of the domain, (b) the simulated hydraulic head contour, and (c) the simulated hydraulic head along the fracture

\subsection{Fracture-matrix flux exchange normal to fractures}

No fracture-matrix flux exchange occurs for impermeable rock matrices or 
impermeable fractures (i.e., sealed fractures, which are simulated in Section 4.1). However, in real cases with a permeable rock matrix and permeable fractures, fracture-matrix flux exchange could be dramatic and should be considered in a rigorous analysis. The fracture-matrix flux exchange or the fluid flow in the direction normal to fractures is basically governed by Equation (4). A facture that is bounded by its two surfaces from the surrounding rock is explicitly represented by these two surface lines, which belong to different physical covers and elements. We develop a new approach that considers the flux across the two surfaces to examine this flux exchange with the surrounding rock, as shown in Figure 8. The directions of the arrows represent the direction of the fluid flow, which is not necessarily toward the fractures. The fractures are assumed to be thin and unfilled, so the distribution of the hydraulic head on each surface of a fracture and within a fracture is uniform in the direction normal to the fracture surfaces. Therefore, we consider these two fracture surfaces to consist of two Dirichlet boundaries, i.e., (1) a Dirichlet boundary with a given hydraulic head $h_{0}=h^{r}(x, y)$ for a fracture with an unknown hydraulic head $h^{f}(\mathrm{x}$, y):

$$
W_{n}^{f r}=-\gamma\left(\int_{\Gamma} k_{f n} \frac{\partial h^{f}}{\partial n}\left(h^{f}-h^{r}\right) d s\right.
$$

Because $h^{f}(x, y)=h^{r_{1}}(x, y)$, Equation (11) could be further expressed as

$$
W_{n}^{f r}=-\gamma\left(\int_{\Gamma} k_{f n} \frac{\partial h^{r^{\prime}}}{\partial n}\left(h^{r^{\prime}}-h^{r}\right) d s\right.
$$

and (2) a Dirichlet boundary with a given hydraulic head $h_{0}=h^{r_{1}}(x, y)$ for a fracture with an unknown hydraulic head $h^{f}(x, y)$ :

$$
W_{n}^{f r^{\prime}}=-\gamma\left(\int_{\Gamma} k_{f n} \frac{\partial h^{f}}{\partial n}\left(h^{f}-h^{r^{\prime}}\right) d s\right.
$$

Because $h^{f}(x, y)=h^{r}(x, y)$, Equation (13) could be further expressed as

$$
W_{n}^{f r^{\prime}}=-\gamma\left(\int_{\Gamma} k_{f n} \frac{\partial h^{r}}{\partial n}\left(h^{r}-h^{r^{\prime}}\right) d s\right.
$$

Combining equations (12) and (14) produces the total work that is associated with normal-to-fracture flow on both surfaces of a fracture:

$$
W_{n}^{f}=-\gamma\left(\int_{\Gamma} k_{f n}\left[\frac{\partial h^{r^{\prime}}}{\partial n}\left(h^{r^{\prime}}-h^{r}\right)+\frac{\partial h^{r}}{\partial n}\left(h^{r}-h^{r^{\prime}}\right)\right] d s\right.
$$

According to the energy-work theorem and the minimization of the total potential energy, we can obtain the final terms in the global equilibrium equations that are associated with normal-to-fracture flow, as shown in Section 3.4. This approach is reasonable because we assume that the fractures are thin and unfilled. If the aperture of a fracture is infinitely large, the surface of the fracture becomes a Dirichlet boundary once the hydraulic head within the fracture is determined. In [21], the authors discussed the relationship between a Dirichlet boundary and a material 
interface. The difference between a material interface and two divided fracture surfaces is that fluid flow occurs in one direction across the material interface but in two opposite directions on the surfaces of fractures, which represents flux exchange with the surrounding rock matrix. Therefore, the expressions for a material interface (derived in [21]) and a fracture (derived in this section) are different and distinguished by the flow directions, even though both the material interface and fracture are assumed to be two connected Dirichlet boundaries.

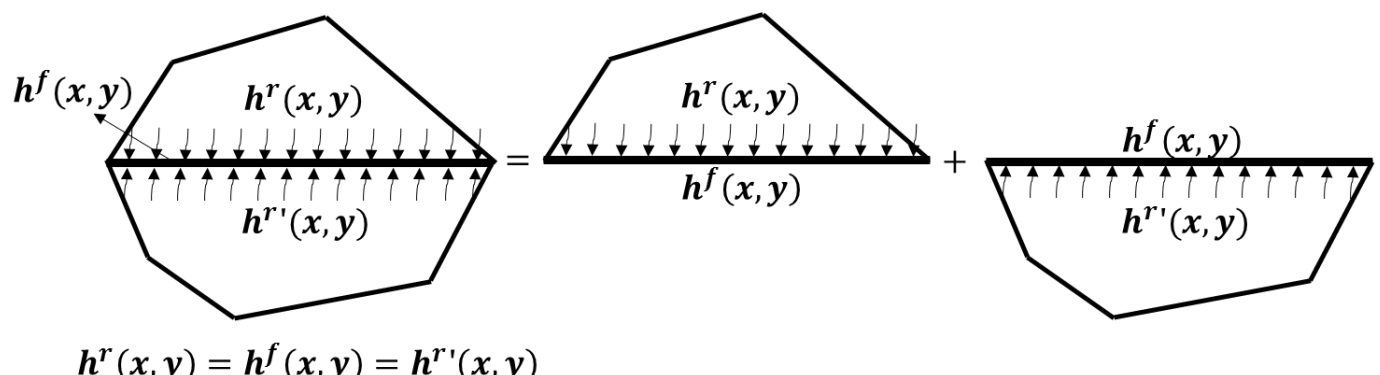

Figure 8 New approach for fracture-matrix flow without introducing DOFs for the fractures

We perform a calculation for a simple $2 \mathrm{~m} \times 2 \mathrm{~m}$ square rock domain that contains a single vertical fracture (Figure 9a) to verify this component for an analysis of the fracture-matrix flux exchange. The upstream and downstream boundaries are the left and right boundaries of the domain, which have hydraulic heads of $10 \mathrm{~m}$ and $0 \mathrm{~m}$, respectively. The permeability contrast between the fracture and rock matrix is 2000 , and the aperture of the fracture is $1 \mathrm{~mm}$. This scenario is a problem that only involves normal flux exchange between a fracture and adjacent rock matrix without along-fracture flow and is equivalent to a fluid flow problem in a single-fracture-included heterogeneous media with three horizontally different types of material (including the fracture), which satisfies Darcy's law. Therefore, the analytical solution for this problem is $h=10-5 x$. We use a minimum mesh with 14 elements and 16 DOFs to calculate this problem and achieve numerical results that exactly agree with the analytical solution, as shown in Figure 9b. The results demonstrate the accuracy and efficiency of this new fracture-matrix flux exchange approach with no need to introduce DOFs for the fractures. For problems with an extremely impermeable rock matrix or extremely impermeable fractures (as in Section 4.1), we can ignore fluid flow in the rock matrix or fractures and perform the calculation by either (1) assigning a small permeability value or (2) deactivating this part in the code because the fracture-matrix flux exchange is negligible. 


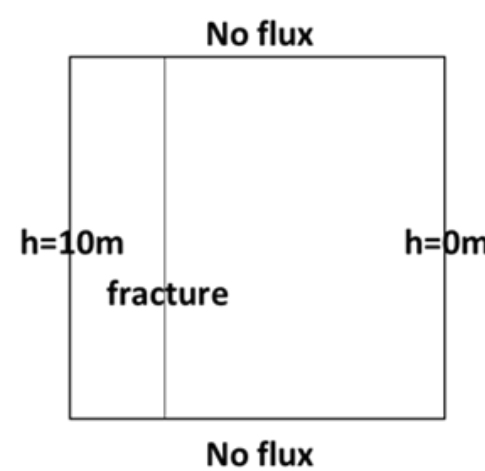

(a)

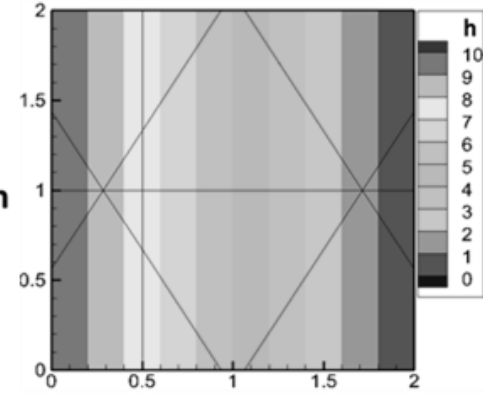

(b)

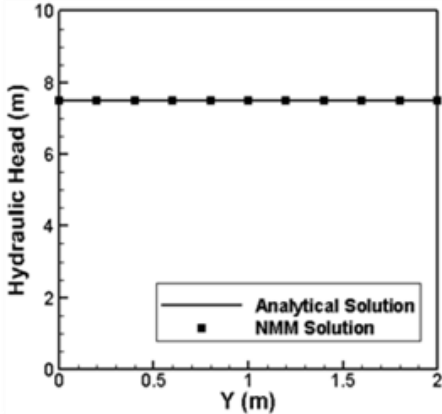

(c)

Figure 9 Verification for normal-to-fracture flow: (a) the geometry and boundary conditions of the domain, (b) the simulated hydraulic head contour, and (c) the simulated hydraulic head along the fracture

\subsection{Dirichlet and Neumann boundary conditions for fractures}

We use a similar method to that for flow in porous media [21] based on a physically verified Lagrange multiplier for fractures that intersect with Dirichlet boundaries. The work that is associated with fracture flow across a Dirichlet boundary is

$$
W_{D}=-\gamma \int_{\Gamma_{D}} k_{f}\left\{\frac{\partial h^{r}(x, y)}{\partial n_{D}}\left[h^{r}(x, y)-h_{0}(x, y)\right]+\frac{\partial h^{r^{\prime}}(x, y)}{\partial n_{D}}\left[h^{r^{\prime}}(x, y)-h_{0}(x, y)\right]\right\} d \Gamma
$$

where $n_{D}$ is the normal direction of the Dirichlet boundary and $\Gamma_{D}$ is the fraction of the Dirichlet boundary within the intersecting fractures.

For fractures that intersect Neumann boundaries, the work according to the energy-work seepage model is

$$
W_{N}=\gamma\left(\int h^{r} \overline{q_{x}} d y+\int h^{r^{\prime}} \overline{q_{x}} d y+\int h^{r} \overline{q_{y}} d x+\int h^{r^{\prime}} \overline{q_{y}} d x\right)
$$

where $W_{N}$ is the work from fluid flow through the Neumann boundaries and $\left(\bar{q}_{x}, \bar{q}_{y}\right)$ are the known flux components.

We can obtain the final terms in the global equilibrium equations for fractures that are subjected to Dirichlet and Neumann boundary conditions according to the energy-work theorem and the minimization of the total potential energy, as shown in Section 3.4 .

The model for fluid flow in porous media was illustrated in [21] in detail. Combined with simplex integration [37], we can solve fluid flow in discrete-fracture porous media in a non-conforming mesh with no need to introduce additional DOFs for the fractures.

\subsection{Establishment of equilibrium equations}

According to the energy-work theorem,

$$
W_{\psi}+\Pi_{\psi}=0
$$

where $\psi$ denotes the work or energy component that is associated with flow in the 
porous media and the boundary conditions in [21] and with the along-fracture flow, normal-to-fracture flow and boundary conditions that were derived in this study.

Combining Equations (3), (12) and (17)-(20) produces the total potential energy $\Pi$. We can derive the global equilibrium equations by minimizing the total potential energy:

$$
\mathbf{C h}=\mathbf{Q}
$$

where $C_{i j}$ are the components of the conductivity matrix $\mathbf{C}$ and $Q_{i}$ is the element of the flux term matrix $\mathbf{Q}$ :

$$
\begin{gathered}
C_{i j}=\frac{\partial^{2} \Pi}{\partial h_{i} \partial h_{j}} \\
Q_{i}=-\frac{\partial \Pi}{\partial h_{i}}
\end{gathered}
$$

The $C_{i j}$ and $Q_{i}$ values from each term in Equations (12) and (17)-(19) combined with Equation (3) are calculated based on simplex integration [37]. Equation (21) shows that no additional DOFs are introduced when using the new model to analyze fracture flow. The accuracy for the along-fracture flow and normal-to-fracture flow was verified by simple examples in sections 3.1 and 3.2, respectively. The entire model package for calculating complicated flow problems in a system with multiple intersecting fractures will be further verified in Section 4.

\section{Model verification}

In this section, we verify the accuracy and efficiency of the new model coded in the NMM package. The model for fractures that intersect with Dirichlet and Neumann boundaries in Section 3.3 is based on a physically verified Lagrange multiplier approach derived in [21] and verified in Section 3.1. The accuracy for the along-fracture flow and normal-to-fracture flow was verified by simple examples with a single fracture in sections 3.1 and 3.2, respectively. Here, we mainly demonstrate the efficiency of mesh generation by the tree-cutting algorithm, the calculation's independency from the mesh orientation, and the accuracy and efficiency for porous media that contain fractures with multiple intersections and different orientations. First, we calculate a problem with impermeable fractures to show the efficiency of mesh generation with the tree-cutting algorithm without activating fluid flow for the fractures. Then, we verify the new model for fracture flow with examples that involve a rotated domain to show the calculation's independency from the mesh orientation, examples that involve several intersecting fractures to show the calculation accuracy for intersecting fractures in porous media, and examples that involve intersecting, inclined fractures to verify the accuracy and efficiency for arbitrarily oriented and intersecting fractures in porous media. We compare the numerical results with available analytical solutions.

\subsection{Example 1: fluid flow in a rock matrix with impermeable fractures}

In some cases, fractures may be sealed and the permeability is extremely low, which 
can be ignored compared to the rock matrix. We calculate such cases in this example to verify the efficiency of the tree-cutting algorithm. In this calculation, the subroutines that are associated with along-fracture flow, normal-to-fracture flow and Dirichlet/Neumann-intersecting-fracture flow are deactivated. Therefore, only flow in the porous media is considered. For this problem, we use the second method to represent fractures in the tree-cutting algorithm (as stated in Section 2.3), i.e., using a single line to represent each fracture while using a relatively dense mesh. This method is appropriate for these cases because only flow in the porous media is considered, and the influence of the fractures on the porous media is as a flow barrier, which is represented by the length of this barrier on a continuous field.

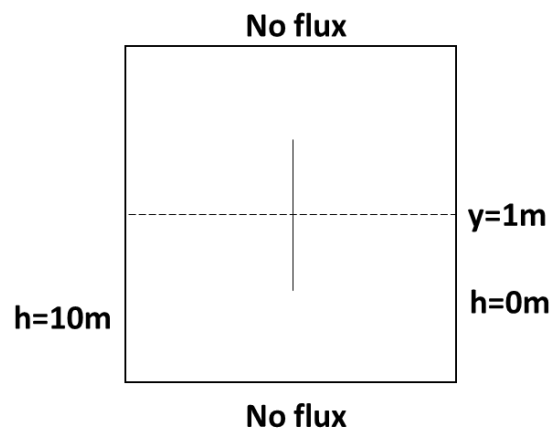

(a)

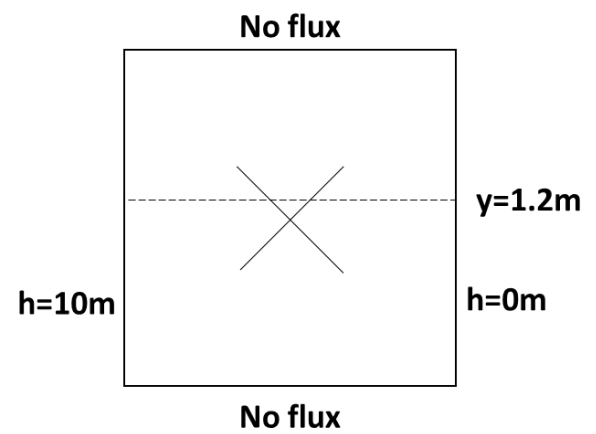

(b)

Figure 10 Geometrical and boundary conditions of the simulated examples with impermeable fractures

We consider two cases. For both cases, the model is a $2 \mathrm{~m} \times 2 \mathrm{~m}$ square rock domain. The upstream and downstream boundaries are the left and right boundaries, which have hydraulic heads of $10 \mathrm{~m}$ and $0 \mathrm{~m}$, respectively. We calculate case 1 with a vertical fracture that is located at $x=1 \mathrm{~m}, \mathrm{y} \in(0.5,1.5)$ and case 2 with two inclined fractures at $y=x$ and $y=2-x, x \in(0.5,1.5)$, as shown in Figure 10.

For case 1, which involves a vertical impermeable fracture, we use 8,20 and 32 layers of elements to calculate and compare the hydraulic head distribution at the profile $\mathrm{y}=1 \mathrm{~m}$, as shown in Figure 11. Although 8 layers of elements can approximately capture the discontinuity in the head gradient, the results deviate at the location of the impermeable fracture from those by 20 and 32 layers of elements. This deviation is caused by a deleted fracture segment from tree cutting when using the second method to represent fractures while using a rather coarse mesh. Meanwhile, 20 layers were sufficient to achieve good results. The abrupt change at $\mathrm{x}=1 \mathrm{~m}$ in Figure $11 \mathrm{~d}$ indicates the impermeability or sealing of the fractures for this case. 


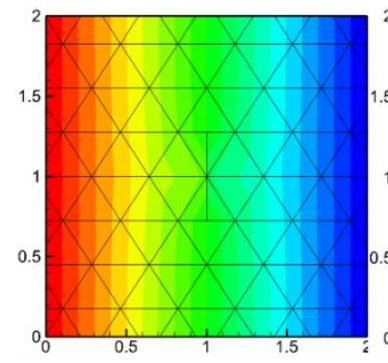

(a)

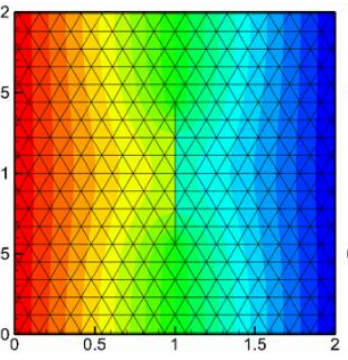

(b)

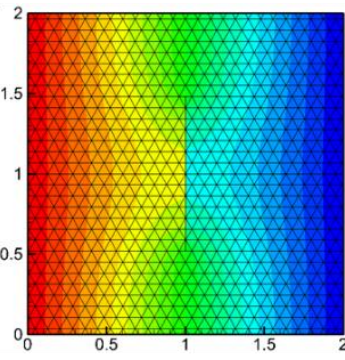

(c)

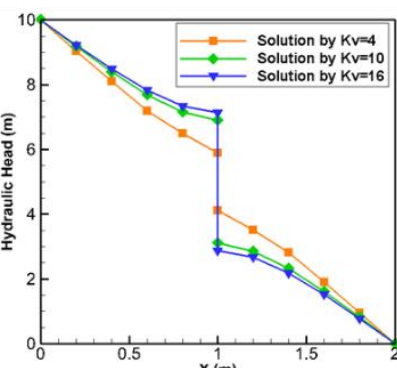

(d)

Figure 11 Simulated results of the hydraulic head distribution with (a) 8 layers of elements, (b) 20 layers of elements, and (c) 32 layers of elements, and (d) a comparison of the hydraulic heads at the profile $y=1 \mathrm{~m}(\mathrm{Kv}$ is the half number of the mesh layers)

For case 2, which involves two intersecting and inclined impermeable fractures, we use 20 and 32 layers of elements to calculate and compare the hydraulic head distribution at the profile $y=1.2 \mathrm{~m}$, as shown in Figure 12. The results with 20 element layers match well with the results with 32 layers because the profile intersects two fractures. Figure $12 \mathrm{c}$ shows the hydraulic head jumps at both intersections.

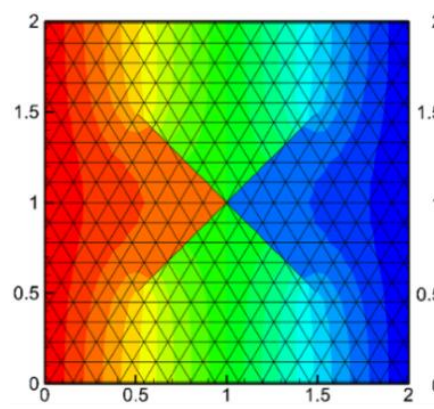

(a)

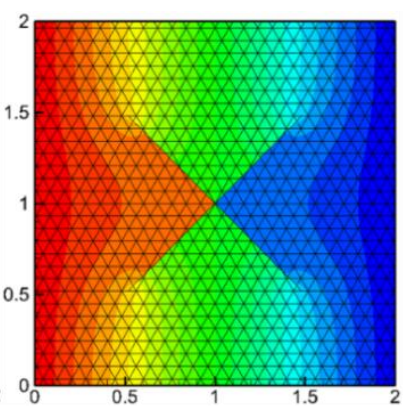

(b)

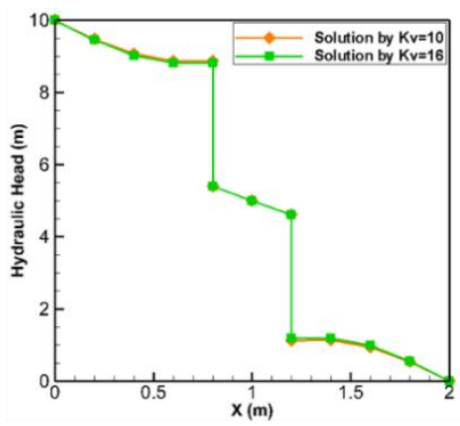

(c)

Figure 12 Simulated results of the hydraulic head distribution with (a) 20 layers of elements and (b) 32 layers of elements, and (c) a comparison of the hydraulic heads at the profile $y=1.2 \mathrm{~m}$

According to this example, the current method is very efficient for modeling flow with multiple impermeable fractures without activating fracture fluid flow. The following examples verify the accuracy and efficiency of the new model for fracture flow.

\subsection{Example 2: fluid flow in a rotated square domain with a single inclined fracture}

In this example, we model a rotated square domain with a single inclined fracture to show the calculation's independency from the mesh orientation. The domain is rotated from the examples in sections 3.1 and 3.2. Their geometry and material properties are the same as those in sections 3.1 and 3.2, as shown in Figure 13. The analytical solution of the hydraulic head retains its linear features from sections 3.1 
and 3.2 after rotation.

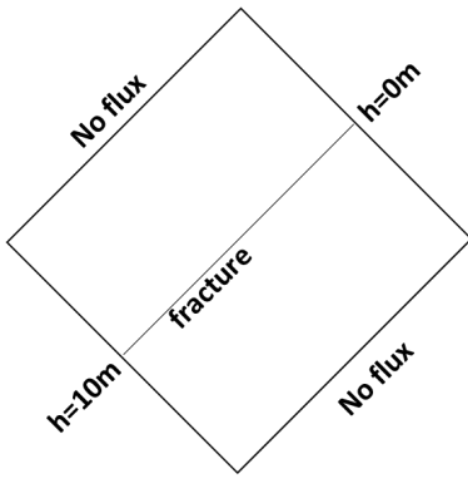

(a)

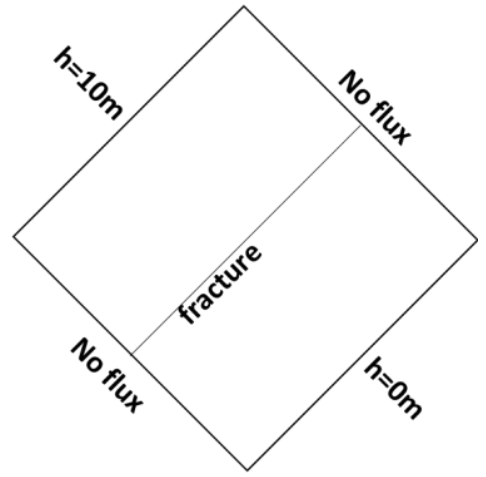

(b)

Figure 13 Geometrical and boundary conditions of the simulated examples

We calculate this problem with a minimal number of elements, and the results in Figure 14 demonstrate the high accuracy and efficiency of the model for problems with inclined fractures. For problems with inclined or geometrically complex boundaries, common numerical methods that require a conforming mesh may need to orient the mesh along the boundaries with a relatively dense mesh or translate the coordinate system. However, in NMM with tree cutting, a coarse regular triangular mathematical mesh, together with the model developed in this study for fracture flow, is sufficiently accurate.

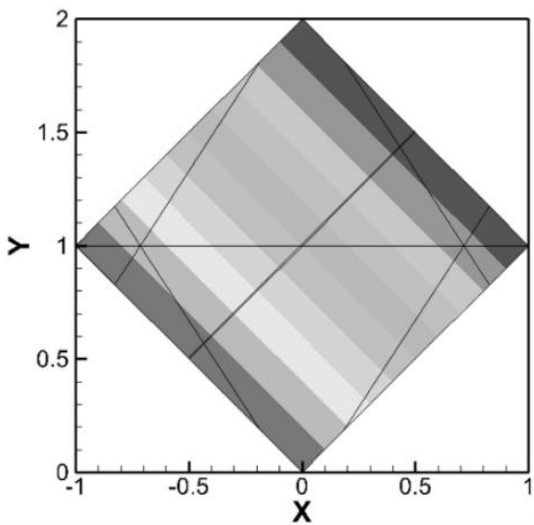

(a)

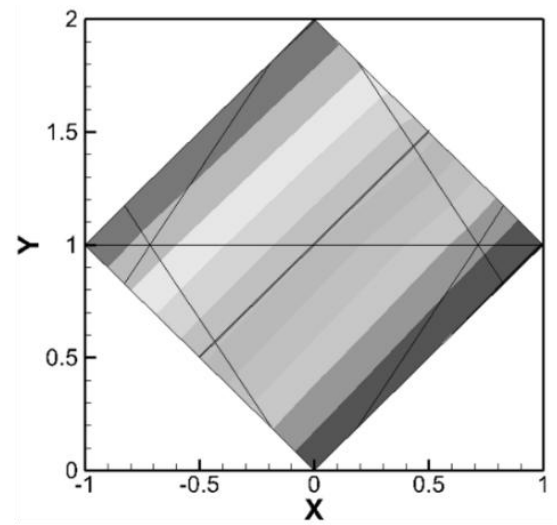

(b)

Figure 14 Simulated results of the hydraulic head distribution

\subsection{Example 3: fluid flow in a rock matrix with two sets of fractures}

In this example, we simulate a rectangular domain with two sets of fractures to verify the accuracy of the model for intersecting fractures. The model boundary conditions are the same as in Section 4.1. The permeability contrast between the rock and fractures is 1:20000. We simulate these cases with 2, 4 and 8 fractures, as shown in Figure 15. The analytical solutions for this problem are the same among all three cases, i.e., $h=10-5 x$. As shown in Figure 16, the results for all three cases with intersecting fractures and different fracture densities achieve analytical solutions. 


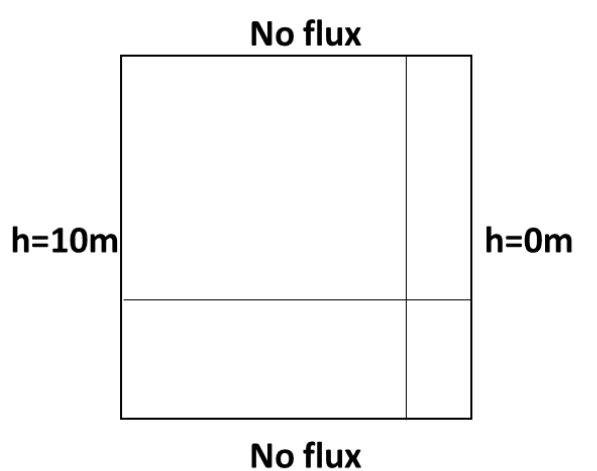

(a)

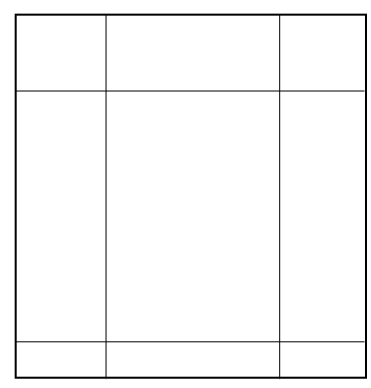

(b)

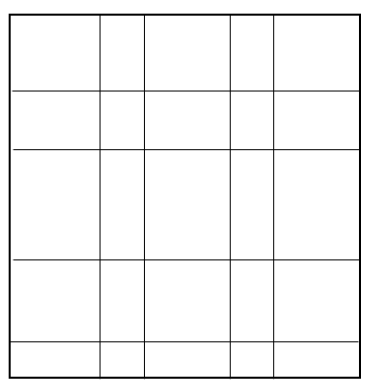

(c)

Figure 15 Geometrical and boundary conditions of the simulated examples

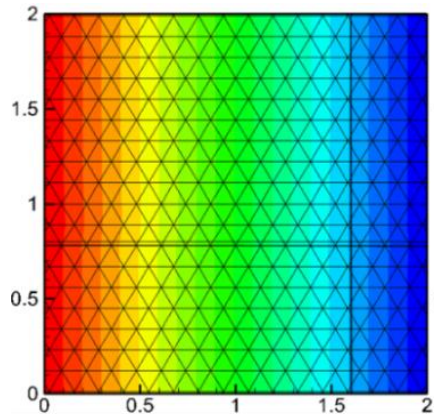

(a)

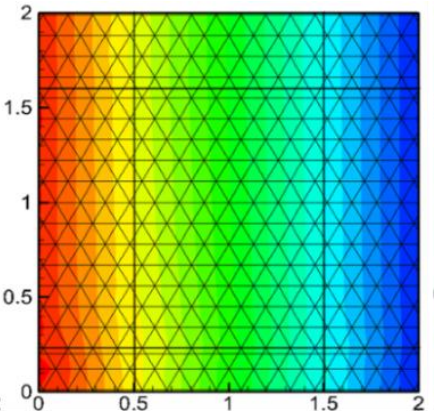

(b)

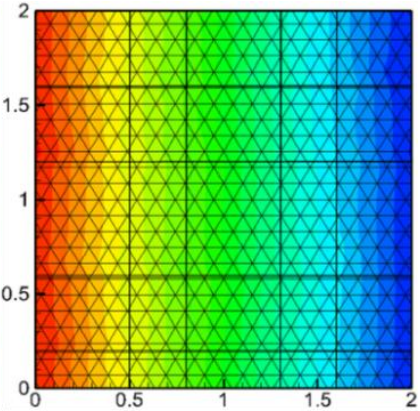

(c)

Figure 16 Simulated results of the hydraulic head distribution

\subsection{Example 4: fluid flow in a rock matrix with inclined fractures}

In this example, we calculate a domain with two sets of inclined fractures to investigate the model for fractures with different orientations, as shown in Figure 17. We simulate cases with 2 and 4 inclined fractures. The permeability contrast between the rock matrix and fractures is 1:100000.

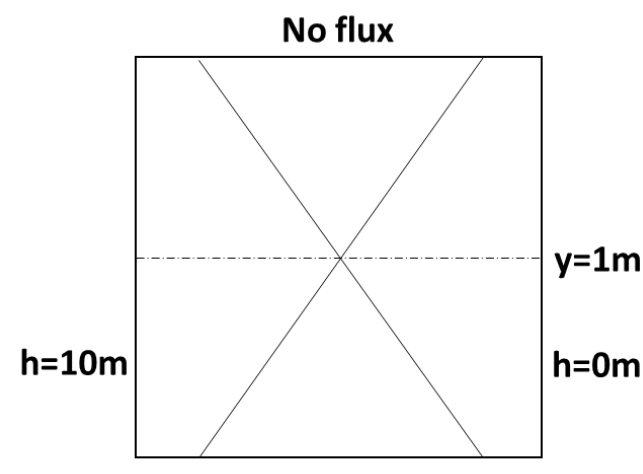

No flux

(a)

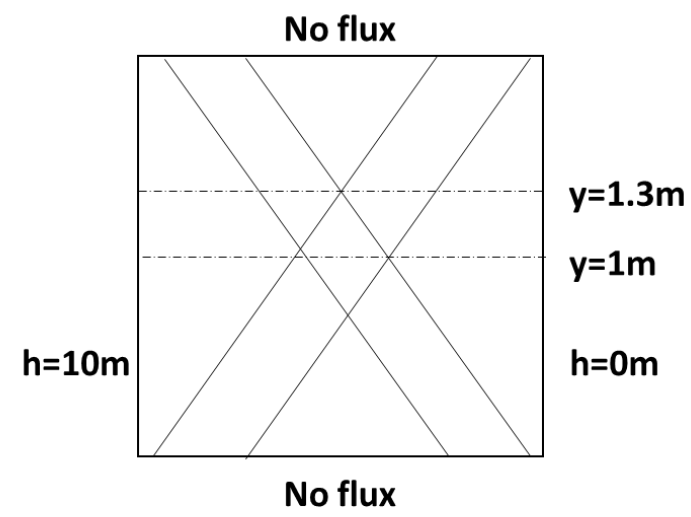

(b)

Figure 17 Geometrical and boundary conditions of the simulated examples

We use 16 and 32 layers of elements to simulate case 1, which involves 2 
inclined fractures, and compare the hydraulic heads at the profile $\mathrm{y}=1 \mathrm{~m}$, as shown in Figure 18. The results from these two mesh sizes agree very well with each other. In Figure 18c, no jump in the hydraulic head occurs at $x=1 \mathrm{~m}$, where the fracture is intersected, indicating the high permeability of the fracture.

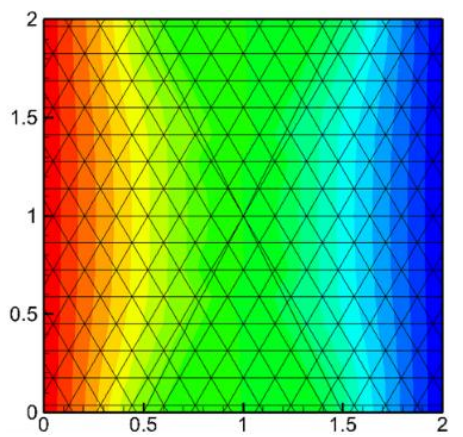

(a)

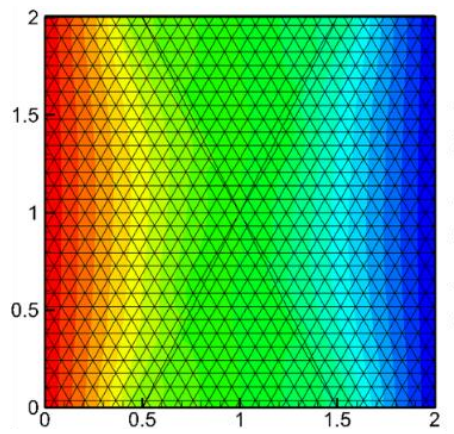

(b)

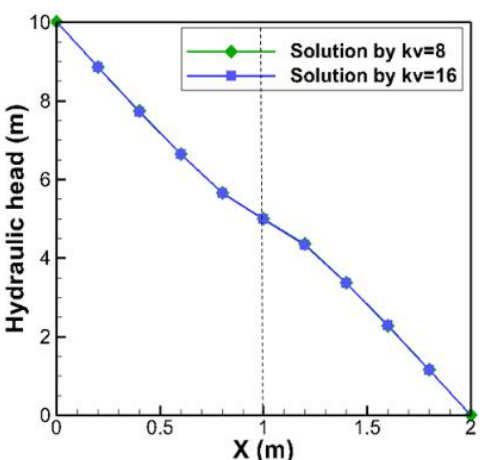

(c)

Figure 18 Simulated results of the hydraulic head distribution with (a) 16 layers of elements and (b) 32 layers of elements, and (c) a comparison of the hydraulic heads at the profile $\mathrm{y}=\mathbf{1} \mathrm{m}$

We use 40 and 50 element layers to calculate case 2, as shown in Figure 19. Their results are consistent, so we only show the contour of the hydraulic head with 50 layers of elements. We also output the hydraulic head that was calculated by the two mesh sizes at the profiles $\mathrm{y}=1 \mathrm{~m}$ and $\mathrm{y}=1.3 \mathrm{~m}$. The profile $\mathrm{y}=1 \mathrm{~m}$ intersects two fractures, and the profile $\mathrm{y}=1.3 \mathrm{~m}$ intersects an intersection of two fractures. No hydraulic head jumps are observed at either intersection, which indicates good accuracy. According to this example and example 3, the new model can model porous rock that contains arbitrarily oriented and intersecting fractures with high permeability contrasts. We can achieve good accuracy with a rather coarse, non-conforming mesh. In the next section, we apply this new model to problems that involve a number of arbitrarily oriented and intersecting fractures.

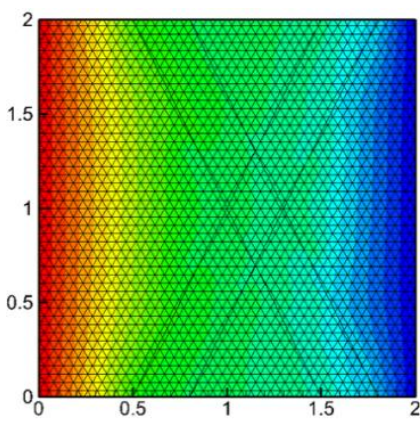

(a)

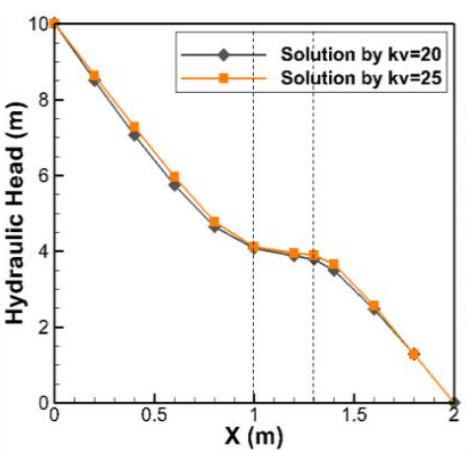

(b)

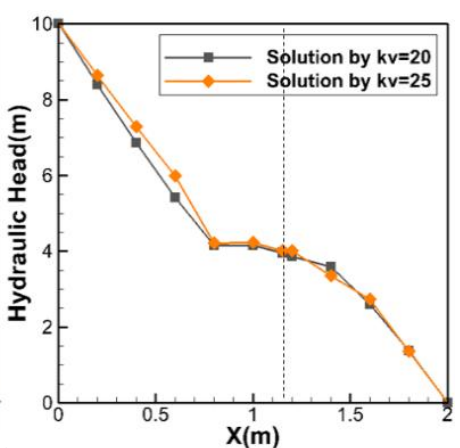

(c)

Figure 19 Simulated results: (a) hydraulic head distribution with 50 layers of elements and a comparison of the hydraulic heads (b) at the profile $y=1 \mathrm{~m}$ and (c) at the profile $y=1.3 \mathrm{~m}$ 


\section{Applications}

As the model was verified step by step including the tree-cutting algorithm, the single-fracture included, multiple-fractures included problems with different orientations, and without dependency on the mesh. Here, we apply the new model to two cases. The first case is a synthetic case that involves a domain with 9 intersecting fractures. The second case involves 100 fractures that were extracted from a discrete fracture network that was generated from mapped fractures at a site in the UK and used in the international DECOVALEX project [47]. In both applications, the fractures are represented by two coinciding lines, as introduced in Section 2.3.

\subsection{Application 1: fluid flow in a domain with 9 fractures}

In this section, we apply our new model to a domain with 9 intersecting fractures. The model geometry, boundary conditions and mesh are shown in Figure 20. The permeability contrast is 1:100000. The simulated results are shown in Figure 21, including the hydraulic head at the profile $y=5 \mathrm{~m}$. This profile intersects with 5 fractures, including a fracture segment that is located at $y=5 \mathrm{~m}, \mathrm{x} \in(2,4.333)$. Figure 21 shows that the hydraulic head in the area along this fracture decreases at a lower rate compared to the surrounding rock, which indicates a more permeable fracture.

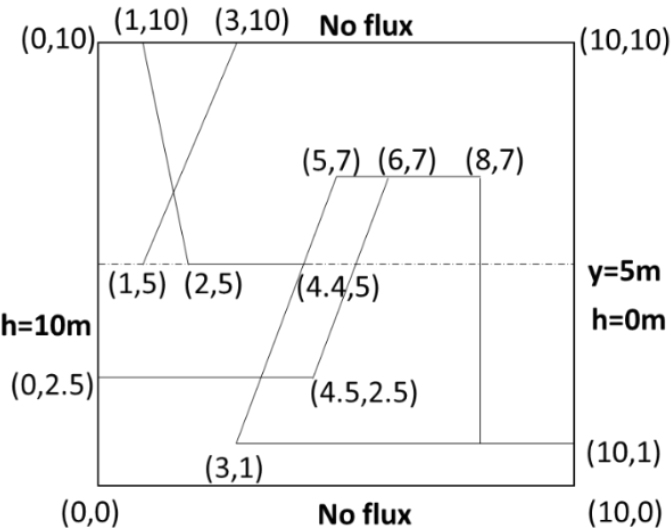

(a)

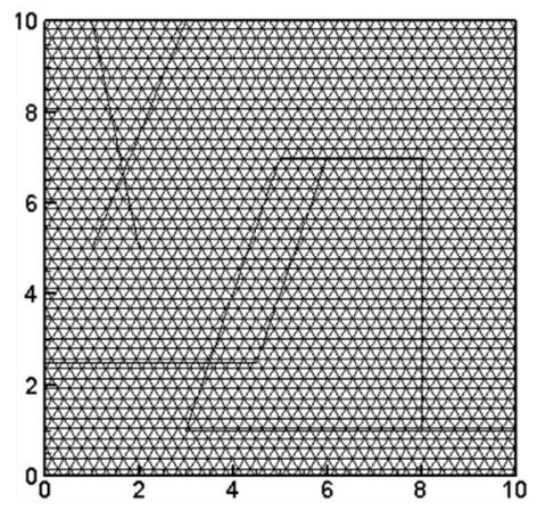

(b)

Figure $\mathbf{2 0}$ (a) Geometrical and boundary conditions and (b) the calculation mesh for a discrete fracture network of 9 fractures

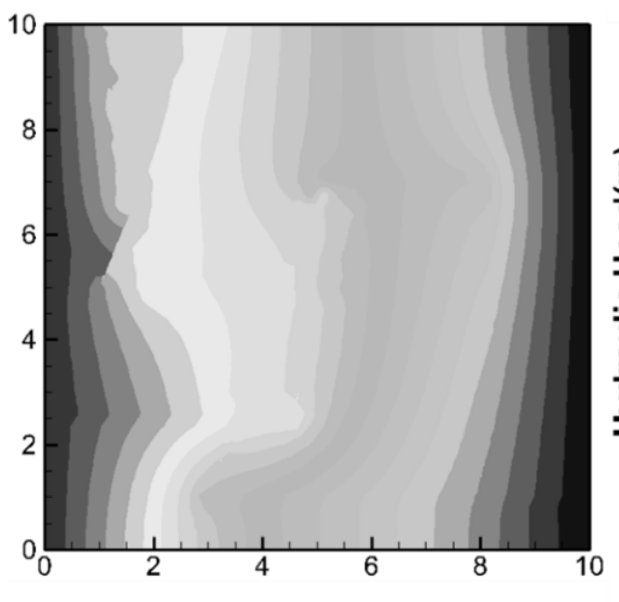

(a)

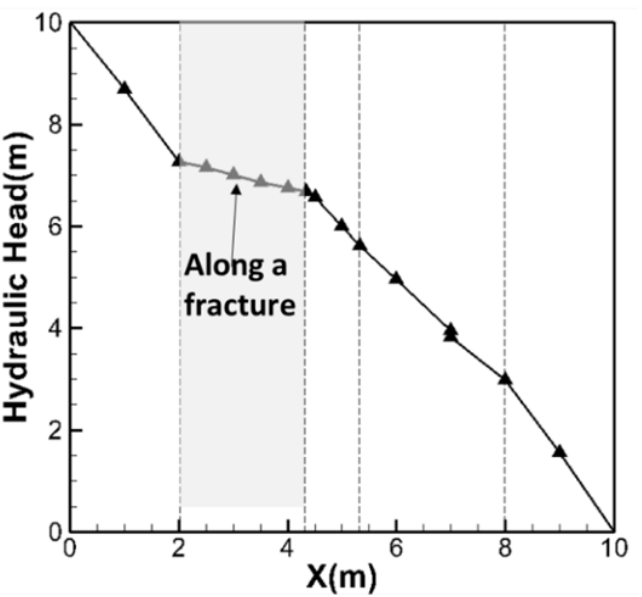

(b) 
Figure 21 (a) Hydraulic head distribution and (b) hydraulic head at the profile $y=5 \mathrm{~m}$ for a discrete fracture network problem with 9 fractures.

\subsection{Application 2: fluid flow in a domain with 100 fractures}

In this application, we apply the new model to solve fluid flow in a $20 \mathrm{~m} \times 20 \mathrm{~m}$ domain with 100 extracted fractures from a real site. The geometric fracture data originated from the Sellafield area, West Cumbria, UK, and have been used as input for several studies for coupled geomechanical-flow-transport analysis in the international DECOALEX project [47]. Originally, the generated fracture network consisted of 7797 individual fractures that were defined in a $40 \mathrm{~m} \times 40 \mathrm{~m}$ domain, with an average fracture spacing of approximately $0.13 \mathrm{~m}$. In the DECOVALEX coupled geomechanical-flow-transport analysis, dual-continuum or dual-porosity models were used for this dense fracture network [47], with the algorithms for calculating the permeability tensor based on Oda's theory. Here, we use a subset of the fractures to create a sparser fracture network that would not be applicable to a dual-continuum model. The generated fracture network is complex, with arbitrarily oriented fractures and various lengths, and we use the new NMM model to calculate the fluid flow and compare the results for different mesh densities.

We extract a $20 \mathrm{~m} \times 20 \mathrm{~m}$ domain that contains 100 fractures with lengths of over $2 \mathrm{~m}$ and calculate the fluid flow. The upstream and downstream boundaries are the left and right boundaries, which have hydraulic heads of $20 \mathrm{~m}$ and $0 \mathrm{~m}$, respectively. Other boundaries are impermeable. The permeability contrast between the rock and fractures is $1: 20$. We use three types of mesh, i.e., a mesh with 60 layers, 76 layers and 80 layers of elements, as shown in Figure 22. Table 2 lists the computation parameters for the three types of mesh. As shown in Figure 23, the overall hydraulic head distributions for these three mesh types match well, although the result with the 60-layer mesh deviated locally from those with the 76-layer mesh and 80-layer mesh. The results with the 76-layer and 80-layer mesh cases agree well with each other, demonstrating a reasonable solution for this complex fracture network problem.

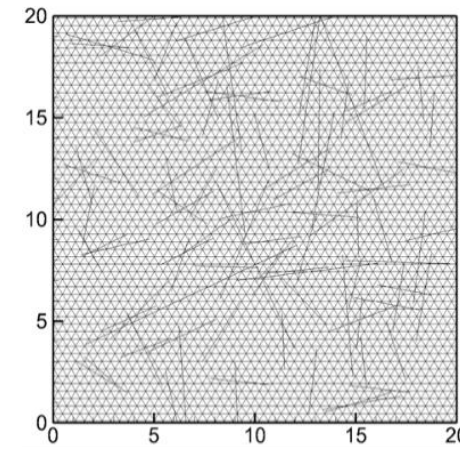

(a)

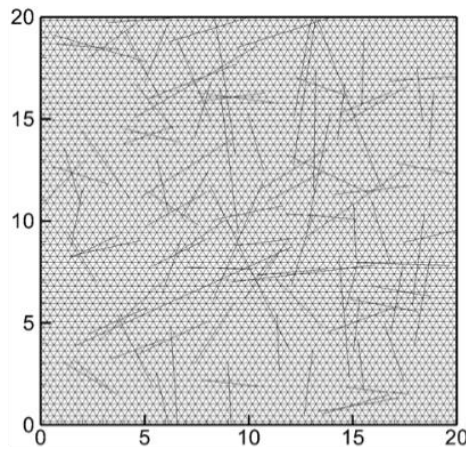

(b)

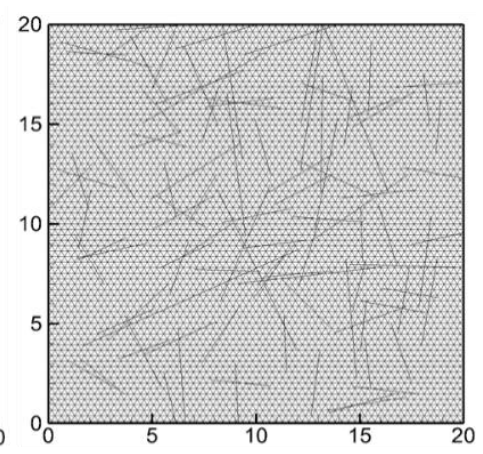

(c)

Figure 22 Simulated problem with 100 fractures when using (a) 60 element layers, (b) 76 element layers and (c) 80 element layers 
Table 2 Computation parameters for application 2 with two different mesh densities

\begin{tabular}{l|ccc}
\hline & 60-layer mesh & 76-layer mesh & 80-layer mesh \\
\hline Number of physical covers & 4741 & 6784 & 7627 \\
Number of elements & 7349 & 10875 & 12109 \\
Number of DOFs & 4741 & 6784 & 7627 \\
\hline
\end{tabular}

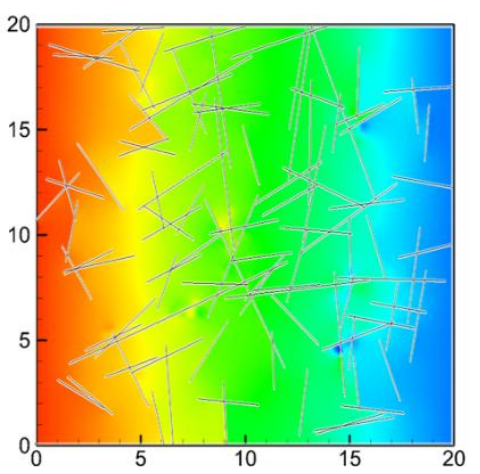

(a)

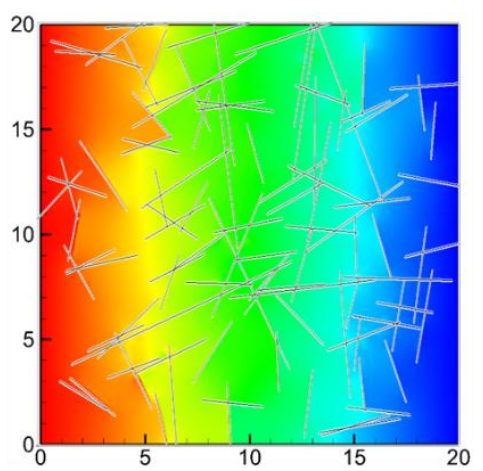

(b)

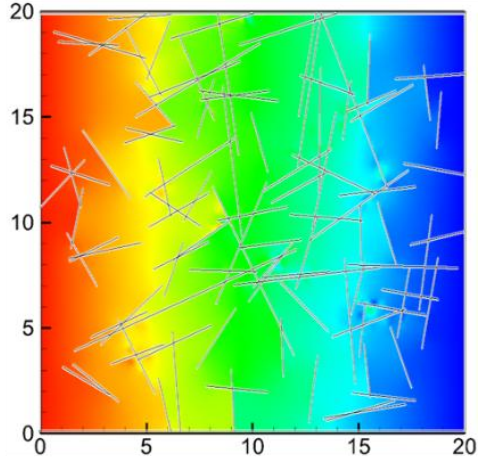

(c)

Figure 23 Simulated hydraulic head results for a problem with 100 fractures when using (a) 60, (b) 76, and (c) 80 layers of elements

\section{Discussion and Perspectives}

In this paper, we developed a 2D NMM model for flow in porous media with discrete fractures. We verified the model step-by-step with various examples, showing that this model can accurately and efficiently analyze flow in permeable porous media that contain arbitrarily oriented and intersecting fractures by using a non-conforming mesh without mesh dependency. The model in its current form can be extended to 3D analysis, with the same routine of tree cutting to generate the mesh, i.e., physical covers and elements. Once the mesh is generated, 3D fluid flow in fractured porous rock can by realized by extending the $2 \mathrm{D}$ model for flow in porous media, as introduced in [21], and the 2D model for flow in fractures developed in this study. Specifically, the approximation should be extended from 2D to 3D, leading to similar formulations for porous media flow as in [21] for 2D analysis. The current model is applicable for 3D analysis of flow in fractures, including both along-fracture flow and normal-to-fracture flow based on its original physical meaning and simplification. The difference is that the fractures are represented by $1 D$ lines in 2D analysis, while the fractures are represented by 2D surfaces in 3D analysis. The components of the work that is associated with the along-fracture flow, fracture-matrix flux exchange, and boundary conditions for fractures can be similarly derived for 2D surfaces, leading to integration on the surfaces instead of integration on line segments for 2D analysis.

The developed model for the analysis of fluid flow in fractured porous media could be a future key component of coupled hydro-mechanical analyses in fractured porous media. As a discontinuous model, the method is suitable for modeling hydro-mechanical behavior, encompassing both continuous and discontinuous 
processes. In addition, this model inherits all the aforementioned advantages of the NMM for discontinuous/continuous modeling for both mechanical and fluid flow problems, as stated in Section 1.

\section{Conclusions}

In this study, we developed an NMM model with a non-conforming mesh to analyze flow in porous media with discrete fractures. Using a two-cover-mesh system in the NMM with physical covers that are divided by fractures and independent approximations that are defined on divided physical covers, it is very convenient to deal with complexly intersecting fractures. A non-conforming mesh is generated with a tree-cutting algorithm, which could be directly used to simulate flow in fractured porous media with an impermeable rock matrix or impermeable fractures. In the tree-cutting algorithm, two alternative methods were suggested to represent fractures to avoid deleting necessary fractures with no or only one intersection from the permeable porous system. We developed a new model to consider fracture flow in the along-fracture and normal-to-fracture directions on this two-cover-mesh system from tree cutting by considering possible boundary conditions with no need to introduce additional DOFs for fractures. In the direction along the fractures, the fracture surfaces belonging to different physical covers conduct flow, which represents fracture-conducting flow. In the direction normal to the fractures, the two fracture surfaces that belong to different physical covers are regarded as Dirichlet boundaries to conduct fluid and to exchange flux with the surrounding rock. Furthermore, the model could consider fractures intersected by Dirichlet or Neumann boundaries.

Combined with a model for fluid flow in porous media [21], we developed a systematic model to analyze flow in discrete-fracture porous media. We designed several simulation examples to verify the efficiency of the tree-cutting algorithm, the calculation's independency from the mesh orientation, and accuracy for porous media that contain fractures with multiple intersections and different orientations. First, we calculated a problem with impermeable fractures to show the efficiency of the mesh generation by the tree-cutting algorithm without activating fluid flow for fractures. Then, we verified the new model for fracture flow with examples that involved a rotated domain, several intersecting fractures and inclined fractures and compared the results with analytical solutions. The good agreement with analytical solutions when using a rather coarse mesh indicated good applicability. According to these verification examples, (1) the current method could very efficiently model flow with multiple impermeable fractures without activating fracture fluid flow; (2) the current model could achieve high accuracy for problems with inclined or geometrically complex boundaries without orienting the mesh along the boundaries with a relatively dense mesh or translating the coordinate system, as in other common numerical methods; and (3) the current model could achieve accurate solutions for intersecting fractures within a rather coarse, non-conforming mesh.

Finally, we applied the model to two cases, i.e., a synthetic case involving a 
domain with 9 intersecting fractures and a case involving 100 fractures that were generated from fractures mapped at a real site, and achieved reasonable results. We showed that the new model is very practical for analyzing fluid flow in porous media with fractures that have a large dimensional contrast under a large hydraulic conductivity contrast. In the future, this model should be coupled with mechanical analysis to analyze the coupled hydro-mechanical behavior in complexly fractured porous media.

\section{Acknowledgments}

The research was supported by the National Natural Science Foundation (No. 51179060) and the Education Ministry Foundation of China (No. 20110094130002) and in part by the U.S. Department of Energy to LBNL under contract No. DE-AC02-05CH11231.

\section{References}

[1] Berkowitz B. Characterizing flow and transport in fractured geological media: a review. Adv Water Resour 2002;25:861-84.

[2] Bonnet E, Bour O, Odling NE, Davy P, Main I, Cowie P, et al. Scaling of fracture systems in geological media. Rev Geophys 2001;39(3):347-83.

[3] MacMinn CW, Szulczewski ML, Juanes R. CO2 migration in saline aquifers. Part 1. Capillary trapping under slope and groundwater flow. J Fluid Mech 2010;662:329-51.

[4] Barker JA. Transport in fractured rock. In: Downing RA, Wilkinson WB, editors. Applied groundwater hydrology. Oxford: Oxford Science Publications, Clarendon Press;1991;199-216.

[5] Neuman SP. Trends, prospects and challenges in quantifying flow and transport through fractured rocks. Hydrogeol J 2005;13:124-47.

[6] Pinder CF, Huyakorn PS, Sudicky EA. Simulation of flow and transport in fractured porous media. In: Bear J, Tsang C-F, de Marsily G, editors. Flow and contaminant transport in fractured rock. San Diego: Academic Press; 1993;395-435.

[7] Sahimi M. Flow and transport in porous media and fractured rock: from classical methods to modern approaches. Weinheim: VCH; 1995.

[8] Barenblatt GI, Zheltov IP, Kochina IN. Basic concepts in the theory of seepage of homogeneous liquids in fissured rocks. Journal of Applied Mathematics and Mechanics 1960; 24(5): 1286-1303.

[9] Pruess K. GMINC- A Mesh Generator for Flow Simulations in Fractured Reservoirs, Lawrence Berkeley Laboratory Report LBL-15227, Berkeley, CA, March 1983.

[10] Adler PM, Thovert J-F. Fractures and fracture networks. Theory and applications of transport in porous media, vol. 15. Boston: Kluwer Academic Publishing; 1999.

[11] Committee on Fracture Characterization and Fluid Flow, US National Committee for Rock Mechanics, Geotechnical Board, Board on Energy and Environmental Systems, Commission on Engineering and Technical Systems, and National Research Council. Rock fractures and fluid flow: contemporary understanding 
and applications. National Academy Press, Washington, DC; 1996.

[12]Zhang Q, Yin J. Solution of two key issues in arbitrary three-dimensional discrete fracture network flow models. Journal of Hydrology 2014;514:281-296.

[13] Berrone S, Fidelibus C, Pieraccini S. Scialo' S. Simulation of the Steady-State Flow in Discrete Fracture Networks with Non-Conforming Meshes and Extended Finite Elements. Rock Mech Rock Eng 2014; 47:2171-2182.

[14] Hyman JD, Karra S, Makedonska N, Gable CW, Painter SL, Viswanathan HS. DFNWORKS: A discrete fracture network framework for modeling subsurface flow and transport. Computers \&Geosciences 2015; 84:10-19.

[15] Belayneh M, Geiger S, Matthai SK. Numerical simulation of water injection into layered fractured carbonate reservoir analogs. AAPG Bull 2006;90(10):1473-93.

[16] Caillabet $Y$, Fabrie P, Landereau P, Noetinger B, Quintard M. Implementation of a finite-volume method for the determination of effective parameters in fissured porous media. Numer Methods Part DE 2000;16:237-63.

[17] Kim JG, Deo MD. Finite element discrete-fracture model for multiphase flow in porous media. AIChE J 2000;46(6):1120-30.

[18] Pruess K, Wang JSY, Tsang YW. On thermohydrologic conditions near high-level nuclear wastes emplaced in partially saturated fractured tuff. 1. Simulation studies with explicit consideration of fracture effects. Water Resour Res 1990;26(6):1235-48.

[19] Reichenberger $V$, Jakobs $H$, Bastian P, Helmig R. A mixed-dimensional finite volume method for two-phase flow in fractured porous media. Adv Water Resour 2006;29(7):1020-36.

[20] Teimoori A, Chen Z, Rahman SS, Tran T. Effective permeability calculation using boundary element method in naturally fractured reservoirs. Petrol Sci Technol 2005;23:693-709.

[21] Hu M, Wang Y, Rutqvist J. On continuous and discontinuous approaches for modeling groundwater flow in heterogeneous media using the Numerical Manifold Method: Model development and comparison. Advances in Water Resources 2015;80:17-29.

[22] Hoteit H, Firoozabadi A. Multicomponent fluid flow by discontinuous Galerkin and mixed methods in unfractured and fractured media. Water Resour Res 2005;41: W11412.

[23] Juanes R, Samper J, Molinero J. A general and efficient formulation of fractures and boundary conditions in the finite element method. Int J Numer Methods Eng 2002;54:1751-1774.

[24] Karimi-Fard M, Durlofsky U, Aziz K. An efficient discrete fracture model applicable for general purpose reservoir simulators. SPE J 2004:227-36.

[25] Alboin C, Jaffre J, Roberts JE, Wang X, Serres C. Domain decomposition for some transmission problems in flow in porous media. Lecture Notes in Physics 2000; 552:22-34.

[26] Angot P, Boyer F, Hubert F. Asymptotic and numerical modelling of flows in fractured porous media. M2AN Math Model Numer Anal 2009;43(2):239-75.

[27] Bogdanov II, Mourzenko VV, Thovert JF, Adler PM. Pressure drawdown well tests 
in fractured porous media. Water Resour Res 2003;39(1):1021.

[28] Bogdanov II, Mourzenko VV, Thovert JF, Adler PM. Effective permeability of fractured porous media in steady state flow. Water Resour Res 2003;39(1):1023.

[29] Bogdanov II, Mourzenko VV, Thovert JF, Adler PM. Effective permeability of fractured porous media with power-law distribution of fracture sizes. PHYSICAL REVIEW E 2007;76:036309.

[30]Bogdanov II, Mourzenko VV, Thovert JF, Adler PM. Two-phase flow through fractured porous media. PHYSICAL REVIEW E 2003;68:026703.

[31] Mourzenko VV, Bogdanov II, Thovert JF, Adler PM. Three-dimensional numerical simulation of single-phase transient compressible flows and well-tests in fractured formations. Mathematics and Computers in Simulation 2011;81:2270-2281

[32] Adler PM, Thovert JF, Mourzenko VV. Fractured Porous Media. Oxford University Press, 2012.

[33] Martin V, Jaffre J, Roberts JE. Modeling fractures and barriers as interfaces for flow in porous media. SIAM J Sci Comput 2005;26(5):1667-91.

[34] Carlo DA, Scotti A. A mixed finite element method for Darcy flow in fractured porous media with non-matching grids. Math Model Numer Anal 2012; 46(02): 465-89.

[35] An X, Fu G, Ma G, A comparison between the NMM and the XFEM in discontinuity modeling, Int. J. Comput. Methods 2012; 9(2): 1240030-1.

[36] Shi GH. Manifold method of material analysis. Trans. 9th Army Conf. on Allied Mathematics and Computing, Minneapolis, USA, 1991: 57-76.

[37] Shi GH. Simplex integration for manifold method, FEM and DDA. Discontinuous Deformation Analysis (DDA) and Simulations of Discontinuous Media. TSI press. 1996: 205-262.

[38] Ma GW, An XM, He L. The numerical manifold method: a review. International Journal of Computational Methods 2010; 7(1): 1-32.

[39] Ma GW, An XM, Zhang HH, Li LX. Modeling complex crack problems with numerical manifold method. International Journal of Fracture 2009; 156(1): 21-35.

[40] Zheng $H, X u$ D. New strategies for some issues of numerical manifold method in simulation of crack propagation. Int. J. Numer. Meth. Engng 2014; 97: 986-1010.

[41] Shi GH. Discontinuous deformation analysis - a new numerical model for the statics and dynamics of block systems. PhD dissertation, University of California, Berkeley, 1988.

[42] Ohnishi Y, Tanaka M, Koyama T, Mutoh K. Manifold method in saturated-unsaturated unsteady groundwater flow analysis. In Third International Conference on Analysis of Discontinuous Deformation---From theory to practice, Vail, Colorado, 3 - 4 January 1999, eds. B. Amadei, 221-230. Alexandria: ARMA.

[43] Wang $Y$, Hu M, Zhou Q, Rutqvist J. Energy-work-based numerical manifold seepage analysis with an efficient scheme to locate the phreatic surface. International Journal for Numerical and Analytical Methods in Geomechanics 
2014; 38: 1633-1650.

[44]Zheng $\mathrm{H}$, LiU F, Li CG. Primal mixed solution to unconfined seepage flow in porous media with numerical manifold method. Applied Mathematical Modelling 2015; 39(2): 794-808.

[45] An XM, Li LX, Ma GW. Prediction of rank deficiency in partition of unity-based methods with plane triangular or quadrilateral meshes. Computer Methods in Applied Mechanics and Engineering 2011; 200: 665-674.

[46] Witherspoon PA, Wang JSY, Iwai K, Gale JE. Validity of the cubic law for fluid flow in a deformable fracture, Water Resour. Res. 1980; 16: 1016-1024.

[47] Rutqvist J, Leung C, Hoch A, Wang Y, Wang Z. Linked multicontinuum and crack tensor approach for modeling of coupled geomechanics, fluid flow and transport in fractured rock. Journal of Rock Mechanics and Geotechnical Engineering 2013;5:18-31. 